\title{
The Torsion of Spinor Connections and Related Structures
}

\author{
Frank KLINKER \\ University of Dortmund, 44221 Dortmund, Germany \\ E-mail: frank.klinker@math.uni-dortmund.de
}

Received August 25, 2006, in final form November 03, 2006; Published online November 09, 2006

Original article is available at http://www.emis.de/journals/SIGMA/2006/Paper077/

\begin{abstract}
In this text we introduce the torsion of spinor connections. In terms of the torsion we give conditions on a spinor connection to produce Killing vector fields. We relate the Bianchi type identities for the torsion of spinor connections with Jacobi identities for vector fields on supermanifolds. Furthermore, we discuss applications of this notion of torsion.
\end{abstract}

Key words: spinor connection; torsion; Killing vector; supermanifold

2000 Mathematics Subject Classification: 17B66; 53C27; 53B20

\section{Introduction}

In this article we introduce the torsion of arbitrary spinor connections. Although the construction depends on additional data on the spinor bundle, namely a choice of charge conjugation, the notion of torsion of a spinor connection is a natural extension of what is usually known as the torsion of a connection on a manifold. In Section 3 we give the relevant definitions and discuss certain properties. In particular, in Proposition 4 we list Bianchi-type identities which connect the torsion and the curvature of the given spinor connection.

The spinor connections for which parallel spinors leads to infinitesimal transformations of the underlying manifold are discussed in Section 4. This turns out to be a symmetry condition on the torsion and lead to the definition of admissibility. In the case of metric connections, admissibility recovers the connections with totally skew symmetric torsion. The latter have been discussed in detail during the last years, e.g. [18] and references therein. Beside these metric connections there are a lot of examples coming from supergravity models and we emphasize on them, e.g. [15] for the basic one. In view of the Fierz relation we formulate the admissibility condition in terms of forms. In Theorem 2 and its extension 3 we give a list of all admissible connections, i.e. connections such that the supersymmetry bracket of parallel spinor fields - when identified with the projection from the endomorphisms of the spinor bundle to the one-forms - closes into the space of Killing vector fields without further assumptions. Such connections are always used when we consider supergravity theories and examine the variations of the odd fields. Moreover admissible pairs are one of the basic objects in our current work on natural realizations of supersymmetry on non-flat manifolds. In Section 4.3 we draw a connection to the geometry of a special class of supermanifold. We show that torsion enters naturally into higher order commutators of canonically defined super vector fields. This yields a connection between the graded Jacobi identity on the superalgebra of vector fields and the Bianchi identities derived in Section 3. The motivation for the introduction of supermanifolds and the consideration of the canonical vector field is taken from the constructions in [24] and [25]. The canonical vector field we consider has also been discussed in [30] from another point of view: One of the vector fields is considered as first order opera- 
tor on the bundle of exterior powers of the spin bundle and it is asked when this operator is a differential.

Section 5 is devoted to examples and applications. We introduce three notions of torsion freeness which are motivated by the discussion so far, and we shortly discuss torsion freeness in the case of flat space. Some properties of spinor connections on flat space have recently been discussed in [11]. In Section 5.3 we discuss brane metrics admitting torsion free admissible subsets.

\section{Preliminaries}

We consider the graded manifold $\hat{M}=(M, \Gamma \Lambda S)$. where $M$ denotes a (pseudo) Riemannian spin manifold and $\Lambda S$ the exterior bundle of the spinor bundle $S$. The splitting $\Lambda S=\Lambda_{0} S \oplus \Lambda_{1} S$ into even and odd forms define the even and odd functions on $\hat{M}$. An inclusion of vector fields on the base manifold $M$ and sections of the spinor bundle $S$ into the vector fields on $\hat{M}$ via

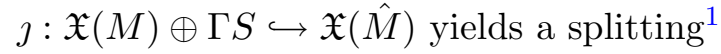

$$
\mathfrak{X}(\hat{M})=\Gamma \Lambda S \otimes \mathfrak{X}(M) \oplus \Gamma \Lambda S \otimes \Gamma S,
$$

compare [26] or [28]. The even and odd parts of the vector fields are given by

$$
\mathfrak{X}(\hat{M})_{\alpha}=\Gamma \Lambda_{\alpha \bmod 2} S \otimes \mathfrak{X}(M) \oplus \Gamma \Lambda_{\alpha+1 \bmod 2} S \otimes \Gamma S, \quad \alpha=0,1 .
$$

The v- and s-like fields are defined by

$$
\mathfrak{X}_{\mathrm{v}}(\hat{M}):=\Gamma \Lambda S \otimes \mathfrak{X}(M), \quad \mathfrak{X}_{\mathrm{s}}(\hat{M}):=\Gamma \Lambda S \otimes \Gamma S .
$$

We call a vector field $X$ of order $(k, 1)$ or $(k, 0)$ if $X \in \Gamma \Lambda^{k} S \otimes \Gamma S$ or $X \in \Gamma \Lambda^{k} S \otimes \mathfrak{X}(M)$, respectively.

The graded manifold $\hat{M}=(M, \Gamma \Lambda S)$ is equipped with a bilinear form $g+C$ where $g$ is the metric on $M$ and $C$ a charge conjugation on $S$. The latter is a spin-invariant bilinear form on $S$. Another important map is the Clifford multiplication

$$
\gamma: \mathfrak{X}(M) \otimes \Gamma S \rightarrow \Gamma S, \quad \gamma(X \otimes \eta)=\gamma(X) \eta=X \eta
$$

with

$$
X Y+Y X=-2 g(X, Y) .
$$

As the notation indicates, we often consider the induced map $\gamma: \mathfrak{X}(M) \rightarrow \Gamma \operatorname{End}(S)$. We call the images of a local frame $\left\{e_{\mu}\right\}$ on $M \gamma$-matrices and write $\gamma\left(e_{\mu}\right)=\gamma_{\mu}$. We always use the abbreviation $\gamma_{\mu_{1} \cdots \mu_{k}}=\gamma_{\left[\mu_{1}\right.} \cdots \gamma_{\left.\mu_{k}\right]}$, e.g. $\gamma_{\mu \nu}=\frac{1}{2}\left(\gamma_{\mu} \gamma_{\nu}-\gamma_{\mu} \gamma_{\nu}\right)$.

The charge conjugation and the Clifford multiplication give rise to the well known morphism $\Gamma S \otimes \Gamma S \hookrightarrow \Lambda \mathfrak{X}(M)$, compare $[25,30,1]$. We denote the projection $\Gamma S \otimes \Gamma S \rightarrow \Lambda^{k} \mathfrak{X}(M)$ by $C_{k}$ and its symmetry by $\Delta_{k} \in\{ \pm 1\}$. The projection is explicitly given by the $k$-form

$$
\left(C_{k}(\phi \otimes \psi)\right)_{\mu_{1} \ldots \mu_{k}}=C\left(\phi, \gamma_{\mu_{1} \cdots \mu_{k}} \psi\right) \text {. }
$$

The symmetry of the morphisms obeys $\Delta_{k}=-\Delta_{k-2}$ and so may be written $\operatorname{as}^{2}$

$$
\Delta_{k}=(-)^{\frac{k(k-1)}{2}} \Delta_{0}^{k+1} \Delta_{1}^{k} .
$$

\footnotetext{
${ }^{1}$ We often use the identifications $\Gamma(E \oplus F)=\gamma E \oplus \Gamma F, \Gamma(E \otimes F)=\Gamma E \otimes_{C^{\infty}(M)} \Gamma F, \Gamma(\operatorname{Hom}(E, F))=$ $\operatorname{Hom}_{C \infty}(\Gamma)(\Gamma E, \Gamma F)$ etc, for sections of vector bundles over the manifold $M$.

${ }^{2}$ This can be made more explicit by evaluating $\Delta_{k}$ for $k=0,1$, compare [25].
} 
The charge conjugations as well as the Clifford multiplication $\gamma: \mathfrak{X}(M) \otimes \Gamma S \rightarrow \Gamma S$ are parallel with respect to the Levi-Civita connection and so are all maps $C_{k}$. The map $\Gamma \operatorname{End}(S) \hookrightarrow \Lambda \mathfrak{X}(M)$ is called Fierz relation and an isomorphism onto the image is explicitly given by

$$
\Omega \mapsto 2^{-\left[\frac{D}{2}\right]} \sum_{n=0}^{\langle D\rangle}(-)^{\frac{n(n-1)}{2}} \frac{1}{n !} \operatorname{tr}\left(\gamma^{(n)} \Omega\right) \gamma_{(n)},
$$

with $\langle\operatorname{dim} M\rangle:=\operatorname{dim} M$ if $\operatorname{dim} M$ is even and $\langle D\rangle:=\frac{1}{2}(\operatorname{dim} M-1)$ if $D$ is odd, compare [32, 23]. If we take into account the charge conjugation to identify $S$ and $S^{*}$ and use (2.2), the Fierz identity is written as

$$
\phi \otimes \psi=\frac{1}{\operatorname{dim} S} \sum_{n} \frac{\Delta_{0}\left(\Delta_{0} \Delta_{1}\right)^{n}}{n !} C\left(\phi, \gamma^{(n)} \psi\right)\left(C \gamma_{(n)}\right) .
$$

We will often use the notations

$$
\{\varphi, \psi\}:=2 C_{1}(\varphi \otimes \psi), \quad\langle\phi, \psi\rangle:=C(\phi, \psi) .
$$

Charge conjugations with $\Delta_{1}=1$ are of special interest, because $\{\cdot, \cdot\}$ may be seen as a supersymmetry bracket in this case. In particular, this choice is possible for Lorentzian space-times, i.e. spin manifold of signature $(-1,1, \ldots, 1)$, compare [25]. Furthermore, we draw the attention to [3] for a classification of bilinear forms also for the case of extended supersymmetry algebras.

Remark 1. Even in the case of $\Delta_{1}=-1$ we may construct a graded manifold with supersymmetry bracket by taking the direct sum of the spinor bundle with itself and provide it with a modified charge conjugation $C \otimes \tau_{2}$. Although there is a choice of charge conjugation with the appropriate symmetry, we are sometimes forced to use the "wrong" one. For example when we want to deal with real spinors. We will discuss such a construction in section 4 so that we will omit it here.

Special vector fields on $\hat{M}$. The charge conjugation $C$ yields an identification $S^{*} \simeq S$. Using this identification a natural inclusion $\jmath: \Gamma S \rightarrow \mathfrak{X}(\hat{M})_{1}$ is given by the interior multiplication of forms and its image is a vector field of degree -1 . Explicitly we have

$$
\jmath(\phi): \Gamma S \rightarrow \Gamma \Lambda S, \quad \jmath(\phi)(\eta)=\langle\phi, \eta\rangle
$$

with the extension as derivation of degree -1 .

Let us consider a connection $D$ on the spinor bundle $S$. For every vector field $X \in \mathfrak{X}(M)$ the action of $D_{X}$ on $\Lambda S$ is of degree zero. This connection gives rise to an inclusion $\jmath_{D}: \mathfrak{X}(M) \rightarrow$ $\mathfrak{X}(\hat{M})_{0}$ given by

$$
\jmath_{D}(X): \Gamma \Lambda S \rightarrow \Gamma \Lambda S, \quad \jmath_{D}(X)(\eta)=D_{X} \eta .
$$

These two inclusions give the natural splitting

$$
\mathfrak{X}(\hat{M})=\Gamma \Lambda S \otimes \mathfrak{X}(M) \oplus \Gamma \Lambda S \otimes \Gamma S .
$$

The endomorphisms of $S$ are vector fields of degree zero on $\hat{M}$ in the natural way. Suppose $\Phi \in \Gamma \operatorname{End}(S) \subset \mathfrak{X}(\hat{M})_{0} \cap \mathfrak{X}_{\mathrm{v}}(\hat{M})$, then the action is given by

$$
\Gamma \Lambda S \supset \Gamma S \ni \eta \longmapsto \Phi(\eta) \in \Gamma S \subset \Gamma \Lambda S .
$$

With respect to a local frame $\left\{\theta_{k}\right\}$ of $S$ the endomorphism $\Phi$ has the components $\Phi_{j}^{i}$ and the associated vector field is given by $\Phi=\Phi_{i}^{j} C^{i k} \theta_{j} \otimes \jmath\left(\theta_{k}\right)$, where $C^{i j} C_{j k}=\delta_{k}^{i}$ and $C_{i j}=C\left(\theta_{i}, \theta_{j}\right)$. 
For $X, Y \in \mathfrak{X}(M), \varphi, \psi \in \Gamma S$ and $\Phi \in \Gamma \operatorname{End}(\Gamma S)$ the following fundamental commutation relations hold:

$$
\begin{aligned}
& {\left[\jmath_{D}(X), \jmath_{D}(Y)\right]=R(X, Y)+\jmath_{D}([X, Y]),} \\
& {[\jmath(\varphi), \jmath(\psi)]=0, \quad\left[\jmath_{D}(X), \jmath(\varphi)\right]=\jmath\left(D_{X}^{C} \phi\right),} \\
& {[\Phi, \jmath(\varphi)]=\jmath\left(-\Phi^{C} \varphi\right), \quad\left[\jmath_{D}(X), \Phi\right]=D_{X} \Phi .}
\end{aligned}
$$

Consider the space $\mathfrak{X}(M) \otimes \Gamma S$ of vector-spinors. The decomposition into irreducible representation spaces yields $\mathfrak{X}(M) \otimes \Gamma S=\Gamma S \oplus \Gamma S_{\frac{3}{2}}$. Using the identification $\mathfrak{X}(M) \simeq \Omega^{1}(M)$ via $g$, the inclusion of $\Gamma S \hookrightarrow \mathfrak{X}(M) \otimes \Gamma S$ is given by the Clifford multiplication $\xi(Y)=Y \xi$. In this way the spin- $\frac{3}{2}$ fields are given by the kernel of the Clifford multiplication. Given a frame $\left\{e_{\mu}\right\}$ on $M$ with associated $\gamma$-matrices $\gamma_{\mu}$ the inclusion is given by

$$
\Gamma S \hookrightarrow \Gamma S \otimes \mathfrak{X}(M), \quad \phi \mapsto(\operatorname{dim} M)^{-1} \gamma^{\mu} \phi \otimes e_{\mu} .
$$

This identification of the spinors in the vector-spinors is used to define a v-like vector field of degree one on the graded manifold. For $\phi \in \Gamma S$ we denote this vector field by $\imath_{D}(\phi)$ and it is defined by the above formula up to the dimension dependent factor together with (2.4):

$$
\imath_{D}(\phi)=\gamma^{\mu} \phi \otimes \jmath_{D}\left(e_{\mu}\right) .
$$

In $[24,25]$ we used this map with $D=\nabla$ the Levi-Civita connection on $M$ and $S$. In [30] this object is considered to construct a (spinor dependent) differential on $\Lambda S$. The action of the differential corresponds to the action of the vector field $\imath_{D}(\phi)$ on the (super)functions $\Gamma \Lambda S$ of $\hat{M}$, i.e.

$$
\Gamma \Lambda S \supset \Gamma S \ni \eta \longmapsto \imath(\phi) \eta=\gamma^{\mu} \phi \wedge D_{\mu} \eta \in \Gamma \Lambda^{2} S \subset \Gamma \Lambda S .
$$

This vector field will be considered in section 4.3.

\section{The torsion of spinor connections}

Given a connection $D$ on $S$ we associate to $D$ the field $\mathcal{A}:=D-\nabla \in \Omega^{1}(M) \otimes \Gamma \operatorname{End}(S)$ where as before $\nabla$ denotes the Levi-Civita connection on $M$. Furthermore, if we denote by $A$ the projection of $\mathcal{A}$ onto the sub algebra which is locally given by $\operatorname{span}\left\{\gamma_{\mu \nu}\right\} \subset\left\{\Phi \in \Gamma \operatorname{End}(S) \mid\left[\Phi, \gamma_{\mu}\right] \subset\right.$ $\operatorname{span}\left\{\gamma_{\nu}\right\}$ for all $\left.\mu\right\}$, then the connection $\nabla^{D}=\nabla+A$ is a metric connection on $M$.

As noted in the last section, the charge conjugation $C: S \rightarrow S^{*}$ as well as the Clifford multiplication $\gamma: T M \otimes S \rightarrow S$ are parallel with respect to the Levi-Civita connection. More precisely we have the following well known result:

Proposition 1. The Clifford multiplication is parallel with respect to the connection $D$ on $S$ and $\widetilde{\nabla}$ on $M$ if and only if $D=\widetilde{\nabla}$ is a metric connection.

The charge conjugation is parallel with respect to the connection $D$ on $S$ if and only if $\mathcal{A}$ takes its values in

$$
\operatorname{span}\left\{\gamma^{\mu_{1} \cdots \mu_{k}} ; \Delta_{k} \Delta_{0}=-1\right\}=\operatorname{span}\left\{\gamma^{\mu_{1} \cdots \mu_{4 k+2}}, \gamma^{\mu_{1} \cdots \mu_{4 k-\Delta_{0} \Delta_{1}}}\right\} .
$$

In particular, $C$ is parallel with respect to every metric connection.

Example 1. In 11-dimensional space-time, i.e. $t=1, s=10$ we have $\Delta_{1}=-\Delta_{0}=1$ so that the map $\Phi \mapsto \Phi^{C}$, with $C\left(\Phi^{C} \eta, \xi\right):=C(\eta, \Phi \xi)$ has (-1)-eigenspace

$$
\operatorname{span}\left\{\gamma^{\mu_{1} \cdots \mu_{4 k+2}}, \gamma^{\mu_{1} \cdots \mu_{4 k+1}}\right\}
$$


and (+1)-eigenspace

$$
\operatorname{span}\left\{\gamma^{\mu_{1} \cdots \mu_{4 k+3}}, \gamma^{\mu_{1} \cdots \mu_{4 k}}\right\} .
$$

In particular, the Clifford multiplication is skew symmetric. For example, consider the supercovariant derivation which come from the supergravity variation of the gravitino and for which $\mathcal{A}_{X}$ has a three-form and a five-form part, compare $[15,14,31,17]$. This connection does not make the charge conjugation parallel.

Due to this example, parallelism of the charge conjugation is not the appropriate notion to be related to supersymmetry in general.

To the connection $D$ on $S$ we will associate another connection $D^{C}$. To construct this we consider the connection $D \otimes \mathbb{1}+\mathbb{1} \otimes D^{C}$ on $S \otimes S$ and the induced connection on $S^{*} \otimes S^{*}$. Then $D \otimes \mathbb{1}+\mathbb{1} \otimes D^{C}$ shall make the charge conjugation parallel, i.e. $\left(D \otimes \mathbb{1}+\mathbb{1} \otimes D^{C}\right) C=0$. For $D=\nabla+\mathcal{A}$ this implies $D^{C}=\nabla-\mathcal{A}^{C}$. The next remark is obtained immediately.

Remark 2. The curvature $R$ of the connection $D$ and the curvature $R^{C}$ of the connection $D^{C}$ are related by $(R(X, Y))^{C}=-R^{C}(X, Y)$.

We endow the bundle of $\operatorname{End}(S)$-valued tensors on $M$ with a connection induced by $D, D^{C}$ and $\nabla$.

Definition 1. Let $\Phi \in \mathfrak{X}(M)^{\otimes k} \otimes \Omega^{1}(M)^{\otimes \ell} \otimes \Gamma \operatorname{End}(S)$. The connection $\hat{D}$ is defined by

$$
\left(\hat{D}_{Z} \Phi\right)(X) \xi:=D_{Z}(\Phi(X) \xi)-\Phi\left(\nabla_{Z} X\right) \xi-\Phi(X) D_{Z}^{C} \xi
$$

for all vector fields $Z, X \in \Omega^{1}(M)^{\otimes k} \otimes \mathfrak{X}(M)^{\otimes \ell}$, and $\xi \in \Gamma S$.

We consider the following ad-type representation of $\operatorname{End}(S)$ on itself.

Definition 2. Let $\Omega \in \operatorname{End}(S)$. We define $\operatorname{ad}_{\Omega}^{C}: \operatorname{End}(S) \rightarrow \operatorname{End}(S)$ by

$$
\operatorname{ad}_{\Omega}^{C} \Phi:=\Omega \Phi+\Phi \Omega^{C} .
$$

This is indeed a representation, because $\operatorname{ad}_{\left[\Omega_{1}, \Omega_{2}\right]}^{C} \Phi=\left[\operatorname{ad}_{\Omega_{1}}^{C}, \operatorname{ad}_{\Omega_{2}}^{C}\right] \Phi$. For $\Omega=\Omega^{+}+\Omega^{-}$, i.e. $\Omega^{C}=\Omega^{+}-\Omega^{-}$we have

$$
\operatorname{ad}_{\Omega}^{C} \Phi=\left[\Omega_{-}, \Phi\right]+\left\{\Omega_{+}, \Phi\right\}
$$

Furthermore we have

$$
\left(\operatorname{ad}_{\Omega}^{C} \Phi\right)^{C}=\left(\Omega \Phi+\Phi \Omega^{C}\right)^{C}=\Phi^{C} \Omega^{C}+\Omega \Phi^{C}=\operatorname{ad}_{\Omega}^{C} \Phi^{C}
$$

which yields

Proposition 2. $\operatorname{ad}_{\Omega}^{C}$ preserves the $( \pm 1)$-eigenspaces of the linear map $\Phi \mapsto \Phi^{C}$ for all $\Omega \in$ $\operatorname{End}(S)$.

Proposition 3. Let $\hat{D}$ be the connection associated to $D$ cf. Definition 1 . Then $D$ and the charge adjoint are compatible in the way that

$$
\hat{D}\left(\operatorname{ad}_{\Omega}^{C} \Psi\right)=\operatorname{ad}_{D \Omega}^{C} \Psi+\operatorname{ad}_{\Omega}^{C} \hat{D} \Psi
$$

for all $\Omega, \Psi \in \Gamma \operatorname{End}(S)$. 
Definition 3. Let $D$ be a connection on the spinor bundle $S$ over the (pseudo) Riemannian manifold $M$ and denote by $\nabla$ the Levi-Civita connection on $M$. The TORsion $\mathcal{T} \in \Omega^{2}(M) \otimes$ $\Gamma \operatorname{End}(S)$ of $D$ is the defined by two times the skew symmetrization of $\hat{D} \gamma: \mathfrak{X}(M) \otimes \mathfrak{X}(M) \rightarrow$ $\Gamma \operatorname{End}(S)$.

\section{Remark 3.}

1. We have $\left(\hat{D}_{X} \gamma\right)(Y)=\hat{D}_{X}(\gamma(Y))-\gamma\left(\nabla_{X} Y\right)$. Using this and $\nabla_{X} Y-\nabla_{Y} X=[X, Y]$ and omitting the map $\gamma$ we may also write

$$
\mathcal{T}(X, Y)=\hat{D}_{X} Y-\hat{D}_{Y} X-[X, Y] .
$$

2. In terms of the difference $\mathcal{A}=D-\nabla \in \Omega^{1}(M) \otimes \Gamma \operatorname{End}(S)$ the torsion may be written as

$$
\mathcal{T}(X, Y)=\operatorname{ad}_{\mathcal{A}(X)}^{C} Y-\operatorname{ad}_{\mathcal{A}(Y)}^{C} X
$$

3. The last point and (3.1) yield that the torsion has symmetry $\Delta_{1}$, i.e. for all $\eta$, $\xi$ we have

$$
C\left(\eta, \mathcal{T}_{\mu \nu} \xi\right)=\Delta_{1} C\left(\xi, \mathcal{T}_{\mu \nu} \eta\right)
$$

4. For a metric connection $D$ on $S$ the torsion is exactly the torsion which is defined by the connection $D$ on the manifold $M$.

The torsion obeys some Bianchi-type identities.

Proposition 4. Let $D$ be a connection on the spinor bundle $S$ over the (pseudo) Riemannian manifold $M$. The torsion $\mathcal{T}$ and the curvature $R$ of $D$ obey

$$
\begin{aligned}
& \hat{D}_{[\kappa} \mathcal{T}_{\mu \nu]}=\operatorname{ad}^{C}\left(R_{[\kappa \mu}\right) \gamma_{\nu]}, \\
& \hat{D}_{[\kappa}\left(\operatorname{ad}_{R}^{C} \gamma\right)_{\mu \nu \rho]}=\operatorname{ad}^{C}\left(R_{[\kappa \mu}\right) \mathcal{T}_{\nu \rho]} .
\end{aligned}
$$

In this context we add the following identity for the curvature $R$ of $D^{3}$ :

$$
D_{[\kappa} R_{\mu \nu]}=0 .
$$

Proof. With Definition 1 the left hand side of (3.3) is given by

$$
\left(\hat{D}_{X} \mathcal{T}\right)(Y, Z) \xi=D_{X}(\mathcal{T}(X, Y) \xi)-\mathcal{T}\left(\nabla_{X} Y, Z\right) \xi-\mathcal{T}\left(Y, \nabla_{X} Z\right) \xi-\mathcal{T}(Y, Z) D_{X}^{C} \xi
$$

We use the definition of the torsion and get

$$
\begin{aligned}
\left(\hat{D}_{X} \mathcal{T}\right)(Y, Z) \xi= & D_{X}(\mathcal{T}(Y, Z) \xi)-\mathcal{T}\left(\nabla_{X} Y, Z\right) \xi-\mathcal{T}\left(Y, \nabla_{X} Z\right) \xi-\mathcal{T}(Y, Z) D_{X}^{C} \xi \\
= & D_{X} D_{Y}(Z \xi)-\underline{D_{X}\left(\nabla_{Y} Z \xi\right)}-\underline{D_{X}\left(Z D_{Y}^{C} \xi\right)}-D_{X} D_{Z}(Y \xi) \\
& +\underline{D_{X}\left(\nabla_{Z} Y \xi\right)}+\underline{D_{X}\left(Y D_{Z}^{C} \xi\right)}-D_{\nabla_{X} Y}(Z \xi)+\nabla_{\nabla_{X} Y} Z \xi \\
& +Z D_{\nabla_{X} Y}^{C} \xi+\underline{D_{Z}\left(\nabla_{X} Y \xi\right)}-\nabla_{Z} \nabla_{X} Y \xi-\underline{\nabla_{X} Y D_{Z}^{C} \xi} \\
& -\underline{D_{Y}\left(\nabla_{X} Z \xi\right)}+\nabla_{Y} \nabla_{X} Z \xi+\underline{\nabla_{X} Z D_{Y}^{C} \xi}+D_{\nabla_{X} Z}(Y \xi) \\
& -\nabla_{\nabla_{X} Z} Y \xi-Y D_{\nabla_{X} Z}^{C} \xi-\underline{D_{Y}\left(Z D_{X}^{C} \xi\right)}+\underline{\nabla_{Y} Z D_{X}^{C} \xi} \\
& +Z D_{Y}^{C} D_{X}^{C} \xi+\underline{D_{Z}\left(Y D_{X}^{C} \xi\right)}-\underline{\nabla_{Z} Y D_{X}^{C}} \xi-Y D_{Z}^{C} D_{X}^{C} \xi .
\end{aligned}
$$

\footnotetext{
${ }^{3}$ This identity holds for any connection $D$ on a vector bundle over $M$, if we endow all tensor bundles with the connection induced by $D$ and the Levi-Civita connection on $M$.
} 
The underlined terms are symmetric with respect to $X, Z$ or $X, Y$. So they vanish when we skew symmetrize the above expression with respect to $X, Y, Z$. So we are left with

$$
\begin{aligned}
& \left(\hat{D}_{X} \mathcal{T}\right)(Y, Z) \xi+\left(\hat{D}_{Z} \mathcal{T}\right)(X, Y) \xi+\left(\hat{D}_{Y} \mathcal{T}\right)(Z, X) \xi \\
& \quad=R(X, Y)(Z \xi)+R(Z, X)(Y \xi)+R(Y, Z)(X \xi)+Z R^{C}(Y, X) \xi+Y R^{C}(X, Z) \xi \\
& \quad+X R^{C}(Y, Z) \xi+\underbrace{\left(R^{0}(Y, X) Z+R^{0}(Z, Y) X+R^{0}(X, Z) Y\right)}_{=0} \xi .
\end{aligned}
$$

With Remark 2, i.e. $R^{C}(Y, X)=R(X, Y)^{C}$, we may rewrite this as

$$
\hat{D}_{[\mu} \mathcal{T}_{\nu \kappa]}=R_{[\mu \nu} \gamma_{\kappa]}+\gamma_{[\kappa}\left(R_{\mu \nu]}\right)^{C}=\operatorname{ad}^{C}\left(R_{[\mu \nu}\right) \gamma_{\kappa]} .
$$

The proof of (3.5) is done by similar calculations. (3.4) follows from (3.2) and (3.5) after skew symmetrization of

$$
\left(\hat{D}_{\kappa} \operatorname{ad}_{R}^{C} \gamma\right)_{\mu \nu \rho}=\operatorname{ad}_{D_{\kappa} R_{\mu \nu}}^{C} \gamma_{\rho}+\operatorname{ad}_{R_{\mu \nu}}^{C} \hat{D}_{\kappa} \gamma_{\rho}
$$

This completes the proof.

Example 2. We consider a manifold which admits geometric Killing spinors. These are spinors which fulfill the equation $\nabla_{X} \phi=-a X \phi$ for a constant $a \neq 0$, the Killing number. This equation has been extensively examined in the literature [8, 29, 21] and in particular [9]. Moreover we would like to stress on [7] where the author draws a remarkable connection between geometric Killing spinors on a manifold and parallel spinors on the cone over the manifold, at least in the Riemannian case.

From the above equation we read that the connection $D$ on the spinor bundle for which the geometric Killing spinors are parallel is given by

$$
D=\nabla+a \cdot \gamma
$$

Suppose $\Delta_{1} \Delta_{0}=-1$, i.e. the Clifford multiplication is skew symmetric. This yields a condition on the connection which will be important in the next section:

$$
\begin{aligned}
\operatorname{ad}^{C}\left(\mathcal{A}_{\{\mu}\right) \gamma_{\nu\}} & =a \gamma_{\{\mu} \gamma_{\nu\}}+a \gamma_{\{\nu} \gamma_{\mu\}}^{C}=-a g_{\mu \nu}+\Delta_{0} \Delta_{1} a \gamma_{\{\nu} \gamma_{\mu\}} \\
& =-a\left(1+\Delta_{1} \Delta_{0}\right) g_{\mu \nu}=0 .
\end{aligned}
$$

The torsion and the curvature of this connection are given by

$$
\mathcal{T}_{\mu \nu}=4 a \gamma_{\mu \nu} \quad \text { and } \quad R_{\mu \nu}=R_{\mu \nu}^{0}+2 a^{2} \gamma_{\mu \nu}
$$

and obey

$$
\hat{D}_{\kappa} \mathcal{T}_{\mu \nu}=-16 a g_{\kappa[\mu} \gamma_{\nu]} \quad \text { and } \quad a d_{R_{\mu \nu}}^{C} \gamma_{\kappa}=R_{\mu \nu \kappa \lambda}^{0} \gamma^{\lambda}+8 a^{2} g_{\kappa[\mu} \gamma_{\nu]}
$$

such that both sides of (3.3) vanish.

\section{Admissible spinor connections}

\subsection{Killing equations and admissible connections}

We examine the conditions on the connection $D=\nabla+\mathcal{A}$, such that the vector field $\{\varphi, \psi\}$ built up by the Killing spinors $D^{C} \varphi=D^{C} \psi=0$ is a Killing vector field, i.e. $\mathcal{L}_{\{\varphi, \psi\}} g=0$. We have

$$
\mathcal{L}_{\{\varphi, \psi\}} g\left(e_{\mu}, e_{\nu}\right)=g\left(\nabla_{\mu}\{\varphi, \psi\}, e_{\nu}\right)+g\left(\nabla_{\nu}\{\varphi, \psi\}, e_{\mu}\right)
$$




$$
\begin{aligned}
& =g\left(\left\{\nabla_{\mu} \varphi, \psi\right\}, e_{\nu}\right)+g\left(\left\{\varphi, \nabla_{\mu} \psi\right\}, e_{\nu}\right)+\{\mu \leftrightarrow \nu\} \\
& =g\left(\left\{\mathcal{A}_{\mu}^{C} \varphi, \psi\right\}, e_{\nu}\right)+g\left(\left\{\varphi, \mathcal{A}_{\mu}^{C} \psi\right\}, e_{\nu}\right)+\{\mu \leftrightarrow \nu\} \\
& =2\left\langle\mathcal{A}_{\mu}^{C} \varphi, \gamma_{\nu} \psi\right\rangle+2\left\langle\varphi, \gamma_{\nu} \mathcal{A}_{\mu}^{C} \psi\right\rangle+\{\mu \leftrightarrow \nu\} \\
& =2\left\langle\varphi, a d_{\mathcal{A}_{\mu}}^{C} \gamma_{\nu} \psi\right\rangle+\{\mu \leftrightarrow \nu\} .
\end{aligned}
$$

This yields

Theorem 1. Let $D$ be a connection on the spinor bundle $S$ over $M$. Suppose $\phi, \psi \in S$ are parallel with respect to the associated connection $D^{C}$. Then the vector field $\{\phi, \psi\}=2 C_{1}(\phi \otimes \psi)$ is a Killing vector field if the symmetric part of $\hat{D} \gamma: \mathfrak{X}(M) \otimes \mathfrak{X}(M) \rightarrow \operatorname{End}(S)$ acts trivially on the parallel spinors. In this case we have

$$
\nabla_{\mu}\{\eta, \xi\}_{\nu}=C\left(\eta, \mathcal{T}_{\mu \nu} \xi\right)
$$

This motivates the next definition.

Definition 4. Let $D$ be a connection on the spinor bundle $S$ over $M$ and $\mathcal{K} \subset \Gamma S$ be a subset.

1. We call $(\mathcal{K}, D)$ admissible if the symmetric part of $\hat{D} \gamma$ acts trivially on $\mathcal{K}$. If $D$ is fixed we call $\mathcal{K}$ admissible.

2. We call $D$ admissible if $\hat{D} \gamma$ is skew symmetric. In this case is $\mathcal{T}=2 \hat{D} \gamma$.

Remark 4. Due to Theorem 1 the admissible subsets of $D^{C}$-parallel spinors are of particular interest.

Example 3. Consider the supergravity connection $D=\nabla+\mathcal{A}$ with $\mathcal{A}=F^{3}+F^{5}$ given by

$$
F^{3}(X)=-\frac{1}{36} X^{\mu} F_{\mu \nu \rho \sigma} \gamma^{\nu \rho \sigma} \quad \text { and } \quad F^{5}(X)=\frac{1}{288} X_{\mu} F_{\nu \rho \sigma \tau} \gamma^{\mu \nu \rho \sigma \tau}
$$

for a 4 -form $F$ on $M$. This connection obeys $F_{\mu}^{5}=-\left(F_{\mu}^{5}\right)^{C}$ and $F_{\mu}^{3}=\left(F_{\mu}^{3}\right)^{C}$ due to Example 1 . Furthermore we have

$$
\operatorname{ad}^{C}\left(\mathcal{A}_{\mu}\right) \gamma_{\nu}=\left[F_{\mu}^{5}, \gamma_{\nu}\right]+\left\{F_{\mu}^{3}, \gamma_{\nu}\right\}=\frac{1}{144} F^{\kappa \rho \sigma \tau} \gamma_{\mu \nu \kappa \rho \sigma \tau}+\frac{1}{9} F_{\mu \nu \kappa \rho} \gamma^{\kappa \rho}
$$

which is indeed skew symmetric with respect to $\mu$ and $\nu$, i.e. the supergravity connection is admissible.

This example can be generalized.

Theorem 2. Let $D$ be a connection on the spinor bundle $S$ of $M$ and $\mathcal{A}:=D-\nabla \in \Omega^{1}(M) \otimes$ $\Gamma \operatorname{End}(S)$. Suppose $\mathcal{A}_{X}$ is homogeneous with respect to $\Gamma \operatorname{End}(S) \simeq \bigoplus_{k} \Omega^{k}(M)$. Consider the decomposition ${ }^{4}$

$$
\Omega^{1}(M) \otimes \Omega^{\ell}(M)=\Omega^{\ell+1}(M) \oplus \Omega^{\ell-1}(M) \oplus \Omega^{(\ell, 1)} .
$$

$\mathcal{A}(X)$ may be written as $\left.\mathcal{A}_{X}=X\right\rfloor F^{\ell+1}+X \wedge G^{\ell-1}+\mathcal{A}_{0}(X)$ with an $(\ell+1)$-form $F$, an $(\ell-1)$-form $G$ and $\mathcal{A}_{0} \in \Omega^{(\ell, 1)}$.

Then $D$ is admissible if and only if $\mathcal{A}_{0}=0$ and $\Delta_{1} \Delta_{\operatorname{deg}}=-1$ or equivalently $\Delta_{0} \Delta_{\operatorname{deg}-1}=$ $(-)^{\operatorname{deg}}$, i.e. $\operatorname{deg} \equiv 3 \bmod 4$, or $1+\Delta_{0} \Delta_{1} \bmod 4$. Here deg denotes the degree of the forms $F$ and $G$ respectively.

${ }^{4} \Omega^{(\ell, 1)}$ denotes the irreducible representation space with highest weight $e_{1}+e_{\ell}$. 
Proof. Consider $\mathcal{A}$ either to be of the form

$$
A_{\mu}=F_{\mu \kappa_{1} \ldots \kappa_{\ell}} \gamma^{\kappa_{1} \ldots \kappa_{\ell}} \quad \text { or } \quad A_{\mu}=G^{\kappa_{1} \ldots \kappa_{\ell-1}} \gamma_{\mu \kappa_{1} \ldots \kappa_{\ell-1}}
$$

with $F \in \Omega^{\ell+1} \oplus \Omega^{(\ell, 1)}$ and $G \in \Omega^{\ell-1}$. In the first case we have

$$
\begin{aligned}
\mathcal{T}_{\mu \nu} & =F_{\mu}{ }^{{ }^{1} \ldots \kappa_{\ell}}\left(\gamma_{\kappa_{1} \ldots \kappa_{\ell}} \gamma_{\nu}+\gamma_{\nu} \gamma_{\kappa_{1} \ldots \kappa_{\ell}}^{C}\right) \\
& =F_{\mu}{ }^{{ }_{1} \ldots \kappa_{\ell}}\left(\gamma_{\kappa_{1} \ldots \kappa_{\ell}} \gamma_{\nu}+\Delta_{0} \Delta_{\ell} \gamma_{\nu} \gamma_{\kappa_{1} \ldots \kappa_{\ell}}\right) \\
& =F_{\mu}{ }^{\kappa_{1} \ldots \kappa_{\ell}}\left(\gamma_{\kappa_{1} \ldots \kappa_{\ell} \nu}+\Delta_{0} \Delta_{\ell} \gamma_{\nu \kappa_{1} \ldots \kappa_{\ell}}\right)-\ell F_{\mu}{ }^{\kappa_{1} \ldots \kappa_{\ell}}\left((-)^{\ell-1}+\Delta_{0} \Delta_{\ell}\right) g_{\nu\left[\kappa_{1}\right.} \gamma_{\left.\kappa_{2} \ldots \kappa_{\ell}\right]} \\
& =F_{\mu}{ }^{{ }{ }_{1} \ldots \kappa_{\ell}}\left(1+(-)^{\ell} \Delta_{0} \Delta_{\ell}\right) \gamma_{\kappa_{1} \ldots \kappa_{\ell} \nu}-\ell F_{\mu \nu}{ }^{\kappa_{2} \ldots \kappa_{\ell}}\left((-)^{\ell-1}+\Delta_{0} \Delta_{\ell}\right) \gamma_{\kappa_{2} \ldots \kappa_{\ell}} .
\end{aligned}
$$

This expression is skew symmetric if and only if $F$ is totally skew symmetric, i.e. $\mathcal{A}_{0}=0$, and $\Delta_{0} \Delta_{\ell}=(-)^{\ell-1}$. With $\operatorname{deg}=\ell+1$ this is exactly the condition stated. The second case is treated in almost the same way.

$$
\begin{aligned}
\mathcal{T}_{\mu \nu} & =G^{\kappa_{1} \ldots \kappa_{\ell-1}}\left(\gamma_{\mu \kappa_{1} \ldots \kappa_{\ell-1}} \gamma_{\nu}+\gamma_{\nu} \gamma_{\mu \kappa_{1} \ldots \kappa_{\ell-1}}^{C}\right) \\
& =G^{\kappa_{1} \ldots \kappa_{\ell-1}}\left(\gamma_{\mu \kappa_{1} \ldots \kappa_{\ell-1}} \gamma_{\nu}+\Delta_{0} \Delta_{\ell} \gamma_{\nu} \gamma_{\mu \kappa_{1} \ldots \kappa_{\ell-1}}\right) \\
& =G^{\kappa_{1} \ldots \kappa_{\ell-1}}\left(\gamma_{\mu \kappa_{1} \ldots \kappa_{\ell-1} \nu}+\Delta_{0} \Delta_{\ell} \gamma_{\nu \mu \kappa_{1} \ldots \kappa_{\ell-1}}\right)-\ell G^{\kappa_{1} \ldots \kappa_{\ell-1}}\left((-)^{\ell-1}+\Delta_{0} \Delta_{\ell}\right) g_{\nu[\mu} \gamma_{\left.\kappa_{1} \ldots \kappa_{\ell-1}\right]} \\
& =G^{\kappa_{1} \ldots \kappa_{\ell-1}}\left((-)^{\ell-1}+\Delta_{0} \Delta_{\ell}\right) \gamma_{\mu \nu \kappa_{1} \ldots \kappa_{\ell}}-\ell G^{\kappa_{1} \ldots \kappa_{\ell-1}}\left((-)^{\ell-1}+\Delta_{0} \Delta_{\ell}\right) g_{\nu[\mu} \gamma_{\left.\kappa_{1} \ldots \kappa_{\ell-1}\right]} .
\end{aligned}
$$

This is skew symmetric if and only if $\Delta_{0} \Delta_{\ell}=(-)^{\ell}$ or $\Delta_{0} \Delta_{\ell-2}=(-)^{\ell-1}$ which with deg $=\ell-1$ finishes the proof.

If $\mathcal{A}$ is of the form $\left.\mathcal{A}_{X}=\hat{\alpha} X \wedge F+\hat{\beta} X\right\rfloor F$ for an $\ell$-form $F$ we may rewrite it as $\mathcal{A}_{X}=$ $\alpha X \cdot F+\beta F \cdot X$ where (.) denotes Clifford multiplication and $\alpha, \beta$ are linear combinations of $\hat{\alpha}, \hat{\beta}$. Therefore, we will restrict ourself often to the two cases $F \cdot X$ and $X \cdot F$.

\section{Remark 5 .}

- To be admissible is a property which has to be checked for every degree of $\operatorname{ad}_{\mathcal{A}}^{C} \gamma$. This yields that the connection $D$ on $S$ is admissible if and only if every homogeneous summand is. Furthermore $D$ is admissible iff $D^{C}$ is admissible, because this fact does only depend on the degree of $\mathcal{A}_{\mu}$ in $\Omega(M)$ which is independent of the charge conjugation.

- For $\left.\mathcal{A}_{X}=X \wedge F+X\right\rfloor G$ admissible the torsion is given by

$$
\mathcal{T}(X, Y)= \pm X \wedge Y \wedge F \pm X\rfloor Y\rfloor G
$$

Example 4. Let $\mathcal{A}(X)$ be of the form $X \wedge F^{(\ell)}$ or $\left.X\right\rfloor F^{(\ell)}$. In eleven dimensional space time this leads to an admissible connection for $\ell=0,3,4,7,8,11$. In Example 3 we have $\left.F_{X}^{3} \sim X\right\rfloor F^{(4)}$ and $F_{X}^{5} \sim X \wedge F^{(4)}$.

Theorems 1 and 2 have an important consequence for metric connections on the spinor bundle.

Corollary 1. Let $D$ be a metric connection on $S . D$ is admissible if and only if $\mathcal{A}_{X}$ is of the form $X\rfloor F^{(3)}$. We write $\mathcal{A}_{\mu}=\frac{1}{4} A_{\mu \nu \kappa} \gamma^{\nu \kappa}$. The torsion tensor in this case is totally skew symmetric and given by $T_{\mu \nu \kappa}=2 A_{[\mu \nu] \kappa}=2 A_{\mu \nu \kappa}$. In other words $D$ is admissible if and only if its torsion is totally skew symmetric.

Metric connections with skew symmetric torsion play an important role in string theory as well as supergravity theories. A lot of literature on this topic has been published during the past few years, see for example [19] or [18] and references therein. 


\subsection{Admissible connections on twisted spinor bundles}

Sometimes it is necessary to introduce $\ell$-form fields which have degree different from those which are allowed by Theorem 2. This is possible in two different ways.

The first way is, in particular, interesting if $M$ is of even dimension $2 n$.

Suppose $n$ is even. In this case the $\ell$-forms with $\ell \equiv 1$ or $1+\Delta_{0} \Delta_{1} \bmod 4$ contribute to an admissible connection by

$$
F^{\nu_{1} \ldots \nu_{\ell}} \gamma_{\nu_{1} \ldots \nu_{\ell}} \gamma_{\mu} \gamma^{*}
$$

This is due to $\Delta\left(\gamma^{(\ell)} \gamma^{*}\right)=\Delta_{2 n-\ell}($ compare (A.3) in Appendix A) and

$$
2 n-1 \equiv 3 \bmod 4, \quad 2 n-3 \equiv 1 \bmod 4, \quad 2 n-\left(1 \pm \Delta_{1} \Delta_{0}\right) \equiv 1 \pm \Delta_{1} \Delta_{0} \bmod 4
$$

for $n$ even as well as Theorem 2 .

If $n$ is odd we have

$$
2 n-1 \equiv 1 \bmod 4, \quad 2 n-3 \equiv 3 \bmod 4, \quad 2 n-\left(1 \pm \Delta_{1} \Delta_{0}\right) \equiv 1 \mp \Delta_{1} \Delta_{0} \bmod 4 .
$$

In this case the $\ell$-form with $\ell \equiv 3$ or $1-\Delta_{0} \Delta_{1} \bmod 4$ contributes cf. (4.1), for the same reason.

The introduction of $\gamma^{*}$ is a bit artificial, because we may express for example $F^{\nu_{1} \ldots \nu_{\ell}} \gamma_{\nu_{1} \ldots \nu_{\ell}}$. $X \gamma^{*}$ as $\pm(* F)^{\nu_{1} \ldots \nu_{2 n-\ell}} \gamma_{\nu_{1} \ldots \nu_{2 n-\ell}} X$. Nevertheless, we will see in Section 5.2 that this is a useful description.

Corollary 2. We consider the projections $\Pi^{ \pm}: S=S^{+} \oplus S^{-} \rightarrow S^{ \pm}$. An $\ell$-form contributes to an admissible connection by $F_{(\ell)} \gamma^{(\ell)} \gamma_{\mu} \Pi^{ \pm}$if and only if $\ell \equiv 3 \bmod 4$ for $n$ odd, or $\ell \equiv 1+\Delta_{0} \Delta_{1} \bmod 4$ for $n$ even.

The second way uses the forms without considering duality, i.e. without adding $\gamma^{*}$. This bypasses the last remark.

We replace the spinor bundle $S$ by the direct sum $S \oplus S$. This space is equipped with a charge conjugation which is given by the charge conjugation $C$ on $S$ twisted by a modified Pauli-matrix, i.e. $C \otimes \tau_{i}$. For $\tau_{0}$ we get the direct sum of $C$ and we denote this usually by $C$, too. The connection $D$ for an $\ell$-form $F$ may be written as

$$
D_{\mu}=\nabla_{\mu}+\frac{1}{\ell !} F_{i_{1} \ldots i_{\ell}} \gamma^{i_{1} \ldots i_{\ell}} \gamma_{\mu} \otimes \tau_{j}=\nabla_{\mu}+F \gamma_{\mu} \tau_{j}
$$

with a matrix $\tau_{j}$. We have

$$
\begin{aligned}
C \otimes \tau_{i}\left(F \gamma_{\mu} \tau_{j} \eta, \gamma_{\nu} \xi\right) & =C\left(F \gamma_{\mu} \tau_{j} \eta, \gamma_{\nu} \tau_{i} \xi\right)=\Delta_{\ell} \Delta_{1} \varepsilon_{j} C\left(\eta, \gamma_{\mu} F \gamma_{\nu} \tau_{j} \tau_{i} \xi\right) \\
& =\Delta_{\ell} \Delta_{1} \varepsilon_{j} \varepsilon_{i j} C \otimes \tau_{i}\left(\eta, \gamma_{\mu} F \gamma_{\nu} \tau_{j} \xi\right)
\end{aligned}
$$

This yields

Theorem 3. For the twisted spinor bundle $S \oplus S$ with charge conjugation $C \otimes \tau_{i}$ the $\ell$-form $F$ contributes to an admissible connection in the form $\frac{1}{\ell !} F_{i_{1} \ldots i_{\ell}} \gamma^{i_{1} \ldots i_{\ell}} \gamma_{\mu} \otimes \tau_{j}$ if and only if

$$
\Delta_{\ell} \Delta_{1} \varepsilon_{j} \varepsilon_{i j}=-1
$$

All possible values for $(\ell, i, j)$ are listed in Table 1.

If we fix $\ell$ we see that the possible values of $j$ depend on the choice of $\tau_{i}$ in the charge conjugation and on $\Delta_{0} \Delta_{1}$ (at least for even $\ell$ ). For $i=j=0$ the two components decouple and we recover the result from Theorem 2 . 
Table 1. Possible choices for $\tau_{j}$ so that the $\ell$-form contributes to an admissible connection if the charge conjugation is given by $C \otimes \tau_{i}$.

\begin{tabular}{c|c|c}
$i$ & $\ell \bmod 4$ & $j$ \\
\hline \hline 0 & 1 & 2 \\
\hline & 3 & $0,1,3$ \\
\hline \hline 1 & 1 & 3 \\
\hline & 3 & $0,1,2$ \\
\hline \hline 2 & 1 & $1,2,3$ \\
\hline & 3 & 0 \\
\hline \hline 3 & 1 & 1 \\
\hline & 3 & $0,2,3$
\end{tabular}

\begin{tabular}{c|c|c}
$i$ & $\ell \bmod 4$ & $j$ \\
\hline \hline 0 & $1-\Delta_{0} \Delta_{1}$ & 2 \\
\hline & $1+\Delta_{0} \Delta_{1}$ & $0,1,3$ \\
\hline \hline 1 & $1-\Delta_{0} \Delta_{1}$ & 3 \\
\hline & $1+\Delta_{0} \Delta_{1}$ & $0,1,2$ \\
\hline \hline 2 & $1-\Delta_{0} \Delta_{1}$ & $1,2,3$ \\
\hline & $1+\Delta_{0} \Delta_{1}$ & 0 \\
\hline \hline 3 & $1-\Delta_{0} \Delta_{1}$ & 1 \\
\hline & $1+\Delta_{0} \Delta_{1}$ & $0,2,3$
\end{tabular}

Remark 6. We draw the attention to the fact that we change the symmetry of $C_{1}$ if we use $\tau_{2}$ to modify the charge conjugation.

\section{Example 5.}

- In [4] the authors discuss pp-wave solutions of type IIA supergravity. The starting point is a Killing equation for the spinors constructed by a 3-form and a 4-form in the following way

$$
D=\nabla+F^{3} \gamma \otimes \tau_{3}+F^{4} \gamma \otimes \tau_{1}
$$

In ten dimensional space time we have two natural ways to choose the charge conjugation $\left(\Delta_{0}=+1\right.$ or -1$)$ and in both cases we have $\Delta_{1}=1$. The above connection is admissible for the choice $\Delta_{0}=-1$ and $i=0$ or 3 as we read from Table 1 .

- In [10] the type IIB supergravity and the variation of its fields are discussed. The vanishing of the gravitino variation leads to a Killing equation where $\mathcal{A}^{C}$ contains all odd $\ell$-forms, $F^{\ell}$ which are twisted by $\tau_{2}$ if the degree is $\ell \equiv 1 \bmod 4$ and by $\tau_{1}$ if the degree is $\ell \equiv 3 \bmod 4$ and furthermore a second three-form, $H^{3}$, twisted by $\tau_{3}$. This is possible only for $i=0$ independent of $\Delta_{0}$.

Moreover the four $\mathbb{Z}_{2}$-symmetries which are given by multiplying the fermion doublets by $\tau_{1}$ or $\tau_{3}$ may be seen as a change of the charge conjugation from $C \otimes \tau_{0}$ to $C \otimes \tau_{1}$ or $C \otimes \tau_{3}$. Now it is evident from Theorem 3 that not all off fields are allowed if we want to keep the connection admissible. In particular, these are $F^{\ell}=0$ for all $\ell$ if $j=1$ and $F^{1}=F^{5}=F^{9}=H^{3}=0$ if $j=3$. These are exactly the truncations which are made in $[10]$.

We carry on considering the supergravity connection cf. [10] which is given by $D^{C}=\nabla-\mathcal{A}^{C}$ with

$$
\begin{aligned}
\mathcal{A}_{\mu}^{C}= & H_{\mu \kappa \lambda} \gamma^{\kappa \lambda}+F_{\kappa}^{1} \gamma^{\kappa} \gamma_{\mu} \otimes \tau_{2}+\frac{1}{3 !} F_{\kappa_{1} \kappa_{2} \kappa_{3}}^{3} \gamma^{\kappa_{1} \kappa_{2} \kappa_{3}} \gamma_{\mu} \otimes \tau_{1}+\frac{1}{5 !} F_{\kappa_{1} \ldots \kappa_{5}}^{5} \gamma^{\kappa_{1} \ldots \kappa_{5}} \gamma_{\mu} \otimes \tau_{2} \\
& +\frac{1}{7 !} F_{\kappa_{1} \ldots \kappa_{7}}^{7} \gamma^{\kappa_{1} \ldots \kappa_{7}} \gamma_{\mu} \otimes \tau_{1}+\frac{1}{9 !} F_{\kappa_{1} \ldots \kappa_{9}}^{9} \gamma^{\kappa_{1} \ldots \kappa_{9}} \gamma_{\mu} \otimes \tau_{2},
\end{aligned}
$$

where $H$ is a torsion three form and the $\ell$-forms are connected by $* F^{1}=F^{9}, * F^{3}=-F^{7}$, and $* F^{5}=F^{5}$. As we mentioned in Example 5 this connection is admissible for the charge conjugation $C \otimes \tau_{0}$. 
Due to the nature of the gravity theories the parallel spinors have a fixed chirality property. More precisely the chirality of the two components of $\eta$ and a relation between both entries, are fixed for all supersymmetry parameters. This may be described by an operator

$$
\mathbb{1} \otimes \tau_{i} \pm \gamma^{*} \otimes \tau_{j}
$$

In the last part of this section we describe admissible connections which are compatible with such chirality property. In contrast to admissibility it is essential to distinguish between $D$ and $D^{C}$, as we will see.

We consider a manifold of even dimension $2 n$ with twisted spinor bundle $S \oplus S$ and charge conjugation $^{5} C \oplus C$. We suppose that the connection $D$ has an admissible contribution of the form

$$
\frac{1}{\ell !} F_{\kappa_{1} \ldots \kappa_{\ell}}^{\ell} \gamma^{\kappa_{1} \ldots \kappa_{\ell}} \gamma_{\mu} \otimes \tau_{i}+\frac{1}{(2 n-\ell) !} F_{\kappa_{1} \ldots \kappa_{2 n-\ell}}^{2 n-\ell} \gamma^{\kappa_{1} \ldots \kappa_{2 n-\ell}} \gamma^{\mu} \otimes \tau_{j}
$$

where the two forms are connected by $* F^{\ell}=w_{\ell} F^{2 n-\ell}$. We insert this as well as (A.2) into the connection and get

$$
\frac{1}{\ell !} F_{\kappa_{1} \ldots \kappa_{\ell}}^{\ell} \gamma^{\kappa_{1} \ldots \kappa_{\ell}} \gamma_{\mu}\left(\mathbb{1} \otimes \tau_{i}-w_{\ell}(-)^{n}(-)^{\frac{\ell(\ell-1)}{2}} \gamma^{*} \otimes \tau_{j}\right)
$$

We define $\Pi_{i j, w}:=\frac{1}{2}\left(\mathbb{1} \otimes \tau_{i}+w \gamma^{*} \otimes \tau_{j}\right)$ which has the following properties:

\section{Lemma 1.}

1. $\Pi_{i j, w}$ has eigenvalue zero if $(i, j)$ is none of the pairs $(0,2),(2,0),(1,3)$, or $(3,1)$ and in the latter cases we have $\Pi_{02, w}^{2}=\frac{1}{2} w \gamma^{*} \otimes \tau_{2}$ and $\Pi_{13, w}^{2}=\frac{1}{2} \mathbb{1}$.

2. The dimension of the zero eigenspace is $\operatorname{dim}\left(\operatorname{ker} \Pi_{i j, w}\right)=\operatorname{dim} S$.

3. For the operators with eigenvalue zero we have $\Pi_{i j, w} \Pi_{i j,-w}=0$ if $i=j$ or $i j=0$ and $\Pi_{12, w} \Pi_{12,-w}=\Pi_{03, w}, \Pi_{23, w} \Pi_{23,-w}=\Pi_{01, w}$ but in all cases

$$
\operatorname{ker} \Pi_{i j, \pm}=\operatorname{im} \Pi_{i j, \mp} .
$$

Proof. The proof is done by taking a look at

$$
\begin{aligned}
& \Pi_{i j, w}^{2}=\frac{\epsilon_{i}+\epsilon_{j}}{4} \mathbb{1}+\frac{1+\epsilon_{i j}}{4} w \gamma^{*} \otimes \tau_{i} \tau_{j}, \\
& \Pi_{i j, w} \Pi_{i j,-w}=\frac{\epsilon_{i}-\epsilon_{j}}{4} \mathbb{1}+\frac{\epsilon_{i j}-1}{4} w \gamma^{*} \otimes \tau_{i} \tau_{j}
\end{aligned}
$$

for the different cases. The kernel of $\Pi_{i j, w}$ which match with the image of $\Pi_{i j,-w}$ is listed in Table 2 .

Due to this lemma we may take $\Pi_{i j, w}$ as a kind of projection which defines the chirality properties of the spinors $\eta \in S \oplus S$. (4.4) with $* F^{\ell}=w_{\ell} F^{2 n-\ell}$ contributes non trivially to an admissible connection in case of a chirality property of the form $\Pi_{k l, w} \eta=0$ if and only if $\operatorname{ker} \Pi_{k l, w} \cap \operatorname{ker} \Pi_{i j, \alpha} \neq 0$ for $\alpha=w_{\ell}(-)^{n}(-)^{\frac{\ell(\ell-1)}{2}}$.

This is the case in (4.2) where all projections have the same image $i m \Pi_{11,-}=i \mathrm{im} \Pi_{22,-}=$ $S^{+} \oplus S^{+} \subset S \oplus S$.

We now ask in what way this chirality operator is transferred to the torsion. The observation which is summarized in the next proposition will, in particular, be used in Section 5.1.

\footnotetext{
${ }^{5}$ We restrict ourself to the case $C \otimes \tau_{0}=C \oplus C$. The other possibilities are treated in the same way.
} 
Table 2. The kernels of $\Pi_{i j, w}$ as subsets of $S \oplus S$.

\begin{tabular}{c|c|c|c}
$\Pi_{00, w}$ & $\Pi_{01, w}$ & $\Pi_{03, w}$ & $\Pi_{11, w}$ \\
\hline$S^{-w} \oplus S^{-w}$ & $\left\{\left(\eta,-w \gamma^{*} \eta\right) \mid \eta \in S\right\}$ & $S^{-w} \oplus S^{w}$ & $S^{-w} \oplus S^{-w}$ \\
\hline \hline$\Pi_{12, w}$ & $\Pi_{22, w}$ & $\Pi_{23, w}$ & $\Pi_{33, w}$ \\
\hline$S^{-w} \oplus S^{w}$ & $S^{-w} \oplus S^{-w}$ & $\left\{\left(\eta, w \gamma^{*} \eta\right) \mid \eta \in S\right\}$ & $S^{-w} \oplus S^{-w}$
\end{tabular}

Proposition 5. Consider a connection D which has a contribution proportional to a projection $c f$. (4.3). Then the associated part of the connection $D^{C}$ as well as the associated part of the torsion of $D$ are proportional to the opposite projection.

Proof. We restrict to the the case $i=j=0$ where the connection has a contribution of the form $\mathcal{A}_{\mu}=F_{(\ell)} \gamma^{(\ell)} \gamma_{\mu} \Pi^{ \pm}$with $\Pi^{ \pm}=\mathbb{1} \pm \gamma^{*}$. The associated part of the connection $D^{C}$ is given by

$$
\begin{aligned}
\mathcal{A}_{\mu}^{C} & =F_{(\ell)}\left(\gamma^{(\ell)} \gamma_{\mu} \Pi^{ \pm}\right)^{C}=F_{(\ell)}\left(\Delta_{1} \Delta_{\ell} \gamma_{\mu} \gamma^{(\ell)} \mp\left(\gamma^{(\ell)} \gamma^{*} \gamma_{\mu}\right)^{C}\right) \\
& =F_{(\ell)}\left(\Delta_{1} \Delta_{\ell} \gamma_{\mu} \gamma^{(\ell)} \mp \Delta_{1} \Delta_{2 n-\ell} \gamma_{\mu} \gamma^{(\ell)} \gamma^{*}\right)=-F_{(\ell)} \gamma_{\mu} \gamma^{(\ell)} \Pi^{\mp},
\end{aligned}
$$

where the last equality is due to the admissibility of the connection. Furthermore we have

$$
\begin{aligned}
\mathcal{T}_{\mu \nu} & =\mathcal{A}_{\mu} \gamma_{\nu}+\gamma^{\nu} \mathcal{A}_{\mu}^{C}=F_{(\ell)}\left(\gamma^{(\ell)} \gamma_{\mu} \Pi^{ \pm} \gamma_{\nu}-\gamma_{\nu} \gamma_{\mu} \gamma^{(\ell)} \Pi^{\mp}\right) \\
& =F_{(\ell)}\left(\gamma^{(\ell)} \gamma_{\mu} \gamma_{\nu}-\gamma_{\nu} \gamma_{\mu} \gamma^{(\ell)}\right) \Pi^{\mp}=F_{(\ell)}\left(\gamma^{(\ell)} \gamma_{\mu \nu}+\gamma_{\mu \nu} \gamma^{(\ell)}\right) \Pi^{\mp} .
\end{aligned}
$$

The proof for $\mathcal{A}_{\mu}=F_{(\ell)} \gamma_{\mu} \gamma^{(\ell)} \Pi^{ \pm}$or $(i, j) \neq(0,0)$ is almost the same.

\subsection{Jacobi versus Bianchi}

In this section we consider a graded manifold of the form $\hat{M}=(M, \Lambda \Gamma S)$ and calculate commutators of the vector fields $\imath(\phi)$ which have been defined in the preliminaries. The (graded) Jacobi identity on the (super) Lie algebra of vector fields will be seen to be related to the Bianchi identities.

We recall the inclusions of the vector fields on $M$, the spinors, and of the endomorphisms of $S$ into the vector fields on $\hat{M}$ as given in Section 2. Due to the fact that we will fix a connection $D$ on $S$, we will drop the index and will write $\jmath: \Gamma S \oplus \mathfrak{X}(M) \rightarrow \mathfrak{X}(\hat{M})$ for the inclusions.

Proposition 6. We consider the graded manifold $(M, \Lambda \Gamma S)$ and a connection $D$ on $S$ which defines the inclusion $\mathrm{\jmath}$ and the map $\imath: \Gamma S \rightarrow \mathfrak{X}(\hat{M})$. Furthermore we consider a linear subspace $\mathcal{K} \subset\left\{\phi \in \Gamma S \mid D^{C} \eta=0\right\}$ such that $(\mathcal{K}, D)$ is admissible. Then the following holds for all $\varphi, \psi \in \mathcal{K}$

$$
[\imath(\varphi), \imath(\psi)]=\mathfrak{B}(R ; \varphi, \psi)+\frac{1}{2} \mathfrak{D}(\mathcal{T} ; \varphi, \psi)
$$

where we use the short notations

$$
\begin{aligned}
& \mathfrak{B}(R ; \varphi, \psi)=\gamma^{\mu} \varphi \wedge \gamma^{\nu} \psi \otimes R_{\mu \nu}, \\
& \mathfrak{D}(\mathcal{T} ; \varphi, \psi)=\left(\gamma^{\mu} \varphi \wedge \mathcal{T}_{\mu \nu} \psi+\gamma^{\mu} \psi \wedge \mathcal{T}_{\mu \nu} \varphi\right) \otimes D^{\nu} . .
\end{aligned}
$$


Proof. For all $\phi, \psi \in \Gamma S$ we have

$$
\begin{aligned}
{[\imath(\phi), \imath(\psi)]=} & \gamma^{\mu} \phi \otimes\left[\jmath\left(e_{\mu}\right), \gamma^{\nu} \psi \otimes \jmath\left(e_{\nu}\right)\right]+\gamma^{\nu} \psi \wedge D_{\nu}\left(\gamma_{\mu} \phi\right) \otimes \jmath\left(e^{\nu}\right) \\
= & \gamma^{\mu} \phi \wedge D_{\mu}\left(\gamma^{\nu} \psi\right) \otimes \jmath\left(e_{\nu}\right)+\gamma^{\mu} \phi \wedge \gamma^{\nu} \psi \otimes\left[\jmath\left(e_{\nu}\right), \jmath\left(e_{\nu}\right)\right] \\
= & \mathfrak{B}(R ; \phi, \psi)+\gamma^{\mu} \phi \wedge \hat{D}_{\mu} \gamma_{\nu} \psi \otimes D^{\nu}+\gamma^{\mu} \psi \wedge \hat{D}_{\mu} \gamma_{\nu} \phi \otimes D^{\nu} \\
& +\gamma^{\mu} \phi \wedge \gamma^{\nu} D_{\mu}^{C} \psi \otimes D_{\nu}+\gamma^{\mu} \psi \wedge \gamma^{\nu} D_{\mu}^{C} \phi \otimes D_{\nu} .
\end{aligned}
$$

In particular, these relations reduce to $(4.5)$ if we restrict to $\mathcal{K}$.

Corollary 3. Consider an admissible metric connection on $S$, i.e. with skew symmetric torsion $T_{\mu \nu \kappa}=2 A_{\mu \nu \kappa}$. In this case (4.5) is given by

$$
[\imath(\varphi), \jmath(\psi)]=\frac{1}{4} R_{\mu \nu \kappa \rho} \gamma^{\mu} \varphi \wedge \gamma^{\nu} \psi \otimes \gamma^{\kappa \rho}+\frac{1}{2} T_{\mu \nu \kappa} \gamma^{\mu} \varphi \wedge \gamma^{\nu} \psi \otimes D^{\kappa}
$$

For the following calculations we restrict to the case that the spinors belong to an admissible subspace $\mathcal{K} \subseteq\left\{\eta \in \Gamma S \mid D^{C} \eta=0\right\}$

$$
\begin{aligned}
{[\imath(\varphi), \mathfrak{B}(R ; \eta, \xi)]=} & \frac{1}{2} \gamma_{\kappa} \varphi \wedge \mathcal{T}^{\kappa \mu} \eta \wedge \gamma^{\nu} \xi \otimes R_{\mu \nu}+\frac{1}{2} \gamma_{\kappa} \varphi \wedge \gamma^{\mu} \eta \wedge \mathcal{T}^{\kappa \nu} \xi \otimes R_{\mu \nu} \\
& -\gamma^{\mu} \eta \wedge \gamma^{\nu} \xi \wedge a d_{R_{\mu \nu}}^{C} \gamma^{\kappa} \varphi \otimes D_{\kappa}+\gamma^{\kappa} \varphi \wedge \gamma^{\mu} \eta \wedge \gamma^{\nu} \xi \otimes\left(D_{\kappa} R\right)_{\mu \nu}, \\
{[\imath(\varphi), \mathfrak{D}(\mathcal{T} ; \eta, \xi)]=} & \frac{1}{2} \gamma^{\kappa} \varphi \wedge \mathcal{T}_{\kappa \mu} \eta \wedge \mathcal{T}^{\mu \nu} \xi \otimes D_{\nu}+\frac{1}{2} \gamma^{\kappa} \varphi \wedge \mathcal{T}_{\kappa \mu} \xi \wedge \mathcal{T}^{\mu \nu} \eta \otimes D_{\nu} \\
& +\frac{1}{2} \mathcal{T}^{\nu \kappa} \varphi \wedge \gamma^{\mu} \eta \wedge \mathcal{T}_{\mu \kappa} \xi \otimes D_{\nu}+\frac{1}{2} \mathcal{T}^{\nu \kappa} \varphi \wedge \gamma^{\mu} \xi \wedge \mathcal{T}_{\mu \kappa} \eta \otimes D_{\nu} \\
& +\gamma^{\kappa} \varphi \wedge \gamma^{\mu} \eta \wedge \hat{D}_{\kappa} \mathcal{T}_{\mu \nu} \xi \otimes D^{\nu}+\gamma^{\kappa} \varphi \wedge \gamma^{\mu} \xi \wedge \hat{D}_{\kappa} \mathcal{T}_{\mu \nu} \eta \otimes D^{\nu} \\
& +\gamma^{\kappa} \varphi \wedge \gamma_{\mu} \eta \wedge \mathcal{T}^{\mu \nu} \xi \otimes R_{\kappa \nu}+\gamma^{\kappa} \varphi \wedge \gamma_{\mu} \xi \wedge \mathcal{T}^{\mu \nu} \eta \otimes R_{\kappa \nu} .
\end{aligned}
$$

From (4.8) and (4.9) we read of the terms of different order in $[\imath(\varphi),[\imath(\eta), \imath(\xi)]]$ :

$$
\begin{gathered}
{[\imath(\varphi),[\imath(\eta), \imath(\xi)]]^{(3,0)}=\frac{1}{4}\left(\gamma^{\kappa} \varphi \wedge \mathcal{T}_{\kappa \mu} \eta \wedge \mathcal{T}^{\mu \nu} \xi+\gamma^{\kappa} \varphi \wedge \mathcal{T}_{\kappa \mu} \xi \wedge \mathcal{T}^{\mu \nu} \eta\right.} \\
\left.+\mathcal{T}^{\nu \kappa} \varphi \wedge \gamma^{\mu} \eta \wedge \mathcal{T}_{\mu \kappa} \xi+\mathcal{T}^{\nu \kappa} \varphi \wedge \gamma^{\mu} \xi \wedge \mathcal{T}_{\mu \kappa} \eta\right) \otimes D_{\nu} \\
+\left(\frac{1}{2} \gamma^{\kappa} \varphi \wedge \gamma^{\mu} \eta \wedge \hat{D}_{\kappa} \mathcal{T}_{\mu \nu} \xi+\frac{1}{2} \gamma^{\kappa} \varphi \wedge \gamma^{\mu} \xi \wedge \hat{D}_{\kappa} \mathcal{T}_{\mu \nu} \eta\right. \\
\left.\quad-\gamma^{\kappa} \eta \wedge \gamma^{\mu} \xi \wedge a d_{R_{\kappa \mu}}^{C} \gamma_{\nu} \varphi\right) \otimes D^{\nu}, \\
{[\imath(\varphi),[\imath(\eta), \imath(\xi)]]^{(4,1)}=\frac{1}{2}\left(\gamma^{\mu} \varphi \wedge \gamma_{\kappa} \eta \wedge \mathcal{T}^{\kappa \nu} \xi+\gamma^{\mu} \varphi \wedge \gamma_{\kappa} \xi \wedge \mathcal{T}^{\kappa \nu} \eta\right.} \\
\left.+\gamma_{\kappa} \varphi \wedge \gamma^{\mu} \xi \wedge \mathcal{T}^{\kappa \nu} \eta+\gamma_{\kappa} \varphi \wedge \gamma^{\mu} \eta \wedge \mathcal{T}^{\kappa \nu} \xi\right) \otimes R_{\mu \nu} \\
+\gamma^{\kappa} \varphi \wedge \gamma^{\mu} \eta \wedge \gamma^{\nu} \xi \otimes\left(D_{\kappa} R\right)_{\mu \nu} .
\end{gathered}
$$

The Jacobi identity, i.e. the vanishing of $\sum_{\varphi, \eta, \xi}[\imath(\varphi),[\imath(\eta), \imath(\xi)]]$ holds independently for the terms of different degree - here £denotes the graded cyclic sum. More precisely:

- The cyclic sums of the first summands in (4.10) and (4.11) vanish due to the symmetry of the involved objects.

- The vanishing of the cyclic sum of the second summand in (4.11) is equivalent to the Bianchi identity (3.5).

- The cyclic sum of the second summand in (4.10) vanishes due to the algebraic Bianchiidentity of the curvature of the Levi-Civita connection. This is due to the following supplement to Proposition 4. 
Lemma 2. Let $\mathcal{K} \subset\left\{\eta \in \Gamma S \mid D^{C} \eta=0\right\}$ such that $(D, \mathcal{K})$ is admissible. Let $\mathcal{T}$ be the torsion of D. Then (3.3) in Proposition 4 reduces to

$$
\left(\hat{D}_{[\kappa} \mathcal{T}_{\mu] \nu}-\operatorname{ad}_{R_{\kappa \mu}}^{C} \gamma_{\nu}-R_{\kappa \mu \nu \lambda}^{0} \gamma^{\lambda}\right) \eta=0
$$

for all $\eta \in \mathcal{K}$.

This yields

Corollary 4. Let $\mathcal{K}, D$ and $\mathcal{T}$ as before. For all spinors $\varphi, \eta, \xi \in \mathcal{K}$ the following holds

$$
\sum_{\varphi, \eta, \xi}\left\{\left(\hat{D}_{[\kappa} \mathcal{T}_{\mu] \nu}-\operatorname{ad}_{R_{\kappa \mu}}^{C} \gamma_{\nu}\right) \xi \wedge \gamma^{\kappa} \varphi \wedge \gamma^{\mu} \eta\right\}=0
$$

Remark 7. As we saw above, the action of $\hat{D}_{[\kappa} \mathcal{T}_{\mu] \nu}-\operatorname{ad}_{R_{\kappa \mu}}^{C} \gamma_{\nu}$ on $\mathcal{K}$ coincides with the action of the curvature of the Levi-Civita connection $R^{0}$ on $\mathcal{K}$. If $D$ is admissible this yields a way to express $R^{0}$ in terms of $R$ and $\mathcal{T}$. Let furthermore $D$ be metric, i.e. a connection with totally skew symmetric torsion. Then the above expression may be written as

$$
R_{\kappa \lambda \mu \nu}^{0}=R_{\kappa \lambda \mu \nu}-D_{[\kappa}^{T} T_{\lambda] \mu \nu}-\frac{1}{4} T_{\kappa \lambda \rho} T_{\mu \nu}^{\rho}-\sigma_{\kappa \lambda \mu \nu}^{T}
$$

with $\sigma_{\kappa \lambda \mu \nu}^{T}=3 T_{\rho[\kappa \lambda} T_{\mu] \nu}{ }^{\rho}$ which is indeed a 4-form. This is due to [16] or [20]. Here $D^{T}$ denotes the connection which differs from $D$ by

$$
\left(D_{X}-D_{X}^{T}\right) T(Y, Z)=\frac{1}{2} T(T(X, Y), Z)+\frac{1}{2} T(Y, T(X, Z)),
$$

i.e. $\left(D_{\mu}-D_{\mu}^{T}\right) T_{\kappa \lambda \nu}=T_{\rho \nu[\lambda} T_{\kappa] \mu}^{\rho}$.

\section{Applications and examples}

\subsection{Torsion freeness}

We consider a connection $D$ on the spinor bundle $S$ and $\mathcal{K} \subseteq \Gamma S$ such that $(D, \mathcal{K})$ is admissible. In (4.5) we defined the map $\mathfrak{D}: S^{2}(\Gamma S) \rightarrow \Lambda^{2} \Gamma S \otimes \mathfrak{X}(M)$ which motivates the following definition.

Definition 5. Let $D$ be a connection on $S$ with torsion $\mathcal{T}$ and $\mathcal{K} \subseteq \Gamma S$ such that $(D, \mathcal{K})$ is admissible.

1. We call $(D, \mathcal{K})$ torsion free if $\mathfrak{D}(\mathcal{T} ; \eta, \xi)=0$ for all $\eta, \xi \in \mathcal{K}$.

2. We call $(D, \mathcal{K})$ strongly torsion free if $\mathcal{T}_{\mu \nu} \eta=0$ for all $\eta \in \mathcal{K}$.

And in view of (4.5)

3. We call $(D, \mathcal{K})$ flat if $\mathfrak{B}(R ; \varphi, \psi)=\mathfrak{D}(\mathcal{T} ; \varphi, \psi)=0$ for all $\varphi, \psi \in \mathcal{K}$.

There are two natural problems: firstly fix $D$ and restrict $\mathcal{K}$ such that one of the properties are obtained, secondly look for conditions on the connection - or the torsion - such that an admissible set $\mathcal{K}$ is "as large as needed".

Of course, admissible subsets $\mathcal{K} \subseteq\left\{\eta \in \Gamma S \mid D^{C} \eta=0\right\}$ will be of particular interest. Due to Theorem 1 the Killing vector fields which we obtain by $\{\mathcal{K}, \mathcal{K}\}$ are parallel with respect to the Levi-Civita connection if $\mathcal{K}$ is strongly torsion free. Therefore, to get non parallel Killing vector fields by $C_{1}$, it is necessary for the connection $D$ on $S$ to admit a part which contribution to the torsion acts non trivially on $\mathcal{K}$. 


\subsubsection{On strongly torsion freeness in $\mathbb{R}^{n}$}

We consider flat $\mathbb{R}^{n}$ with spinor bundle $S$ and connection

$$
D_{X}^{C} \psi=X(\psi)-\mathcal{A}_{\mu}^{C} \psi
$$

where the potential $\vec{A}=\left(\mathcal{A}_{1}^{C}, \ldots, \mathcal{A}_{n}^{C}\right)$ is constructed from forms on $\mathbb{R}^{n}$ with constant coefficients.

Example 6. Consider $\mathbb{R}^{2 n}$ with connection $D_{\mu}^{C}=d_{\mu}-\mathcal{A}_{\mu}^{C}$ on its spinor bundle. Let $\mathcal{A}^{C}$ be determined by a three-form $F$, moreover $F$ shall be a one-form with values in $\mathfrak{s u}(n)$. Then $\mathfrak{h o l} \subset \mathfrak{s u}(n)$ and there exist two parallel pure spinors $\eta, \bar{\eta}$ which are associated via charge conjugation. These spinors obey $\mathfrak{B}(R ; \eta, \eta)=\mathfrak{B}(R, \bar{\eta}, \bar{\eta})=0$. We use the decomposition $\mathbb{C}^{2 n}=\mathbf{n} \oplus \overline{\mathbf{n}}$ where the complex structure obeys $\mathbf{n} \bar{\eta}=\overline{\mathbf{n}} \eta=0$. If $F \in \Lambda^{3} \mathbb{C}^{2 n} \cap(\overline{\mathbf{n}} \otimes \mathfrak{s u}(n))$ the torsion acts trivially on $\eta$. In this case the subspace spanned by this sole odd generator is strongly torsion free, in particular, it would have vanishing center. If $F \in \Lambda^{3} \mathbb{C}^{2 n} \cap(\mathbf{n} \otimes \mathfrak{s u}(n))$ the same holds for $\bar{\eta}$. We emphasize that in both cases the three-form is not real and that for a real three-form a trivial action on one of the spinors is only possible in case of vanishing torsion.

Example 7. Suppose $\mathcal{A}$ is obtained by a constant form and $\mathcal{A}_{X} \propto \Pi^{+}\left(\Pi^{-}\right)$for a projection $\Pi^{ \pm}$ cf. (4.3). Due to Proposition $5 D^{C}$ and $\mathcal{T}$ are proportional to the opposite projection $\Pi^{-}\left(\Pi^{+}\right)$. So $\mathcal{K}$ spanned by the constant positive (negative) spinors is strongly torsion free.

The last example can be generalized to

Remark 8. Strongly torsion freeness can not be achieved by pure chirality considerations due to Proposition 5, when we want to deal with spinors which are not Levi-Civita parallel. In this case strongly torsion freeness leads to new algebraic constraints on the fields.

We will discuss torsion free structures which are not strongly torsion free in Section 5.2 (generalizing Example 6) and 5.3.

\subsubsection{A comment on differentials}

As we mentioned in the introduction and as performed in [30] we may take the vector field $\imath_{D}(\eta)=\gamma^{\mu} \eta \otimes \jmath_{D}\left(e_{\mu}\right)$ as degree-one operator on $\Lambda S$ and look for conditions such that this operator is a differential. We immediately get

Proposition 7. Let $D$ be a connection on a spinor bundle $S$ over the (pseudo) Riemannian manifold $M$. Consider the vector field $\imath(\eta)$ on the graded manifold $(M, \Gamma \Lambda S)$. Let $D^{C} \eta=0$, then $\imath(\eta)$ is a differential on $\Gamma \Lambda S$ if and only if $(D,\{\eta\})$ is flat.

When we consider admissible subspaces $\mathcal{K}$ of order one we have to take the collection of all elements in $\left\{\mathfrak{B}(R ; \eta, \xi) \mid D^{C} \eta=D^{C} \xi=0\right\}$ and $\left\{\mathfrak{D}(\mathcal{T} ; \eta, \xi) \mid D^{C} \eta=D^{C} \xi=0\right\}$ and discuss whether or not these terms vanish. In particular, if the dimension of $\mathcal{K}$ is large the conditions on the torsion are very restrictive. When we consider the differential point of view we only have to discuss the terms $\mathfrak{B}(R ; \eta, \eta)$ and $\mathfrak{D}(\mathcal{T} ; \eta, \eta)$ for one fixed spinorial entry.

In [30] and [25] the condition on $\mathfrak{B}$ is discussed for the untwisted case. The twisted case is touched when the authors discuss real spinors. The main emphasis is on metric connections $D$ of holonomy $\mathfrak{g} \subset \mathfrak{s o}(n) \subset \mathfrak{s l}\left(2^{\left[\frac{n}{2}\right]}\right)$ with $\mathfrak{g}=\mathfrak{s u}\left(\frac{n}{2}\right), \mathfrak{s p}\left(\frac{n}{4}\right), \mathfrak{s p i n}(7)$ if $n=8$, or $\mathfrak{g}_{2}$ if $n=7$. The discussion in [25] is restricted to the torsion free Levi-Civita connection. If we want to cover non-torsion free metric connections - or general spinorial connections - we have to take into account the $\mathfrak{D}$-contribution which yields further restrictions and we recall Example 6 and the examples below. 


\subsection{Parallel pure spinors}

We consider a Riemannian manifold $M$ of even dimension $2 n \geq 4$. Consider a pure spinor $\eta \in \Gamma S$. We will discuss conditions on a connection $D$ such that $\mathfrak{B}(R ; \eta, \eta)$ or $\mathfrak{D}(\mathcal{T} ; \eta, \eta)$ vanish. As before, the case of a $D^{C}$-parallel pure spinor is of particular interest due to Theorem 1 , Section 4.3, and Proposition 7. Although we deal with forms of arbitrary degree, we always specialize to the metric case.

A pure spinor is characterized by the following two equivalent conditions (compare $[13,22])$.

(1) The space $\{X \in T M \mid X \eta=0\}$ has maximal dimension, namely $n$.

(2) $C_{k}(\eta, \eta)=0$ for all $k \neq n$.

Furthermore a pure spinor is either of positive or of negative chirality and the vector field $\{\eta, \eta\}$ vanishes. The symmetry $\Delta_{k}$ and the chirality of $C_{k}$ are given by

\begin{tabular}{c||c|c|c|c}
$2 n \bmod 8$ & 0 & 2 & 4 & 6 \\
\hline \hline$\Delta_{2 m}$ & $(-)^{m}$ & $\pm(-)^{m}$ & $-(-)^{m}$ & $\mp(-)^{m}$ \\
\hline$\Delta_{2 m+1}$ & $\pm(-)^{m}$ & $(-)^{m}$ & $\mp(-)^{m}$ & $-(-)^{m}$ \\
\hline \hline chirality & non chiral & chiral & non chiral & chiral
\end{tabular}

The different signs belong to the choice of charge conjugation. Chiral means $C: S^{ \pm} \otimes S^{\mp} \rightarrow \mathbb{C}$ and non-chiral (nc) means $C: S^{ \pm} \otimes S^{ \pm} \rightarrow \mathbb{C}$. Examining this table yields that the second part 2 in the characterization may be relaxed as follows

$\left(2^{\prime}\right)$ The chiral (or anti-chiral) spinor $\eta$ is pure if $C_{k}(\eta, \eta)=0$ for all $k-n \equiv 0 \bmod 4, k \neq n$.

In particular $C_{n}$ has symmetry $\Delta_{n}=1$ in all cases.

We take a closer look at $\mathfrak{B}(R ; \eta, \eta)=\gamma^{\mu} \eta \wedge \gamma^{\nu} \eta \otimes R_{\mu \nu}$. We use the Fierz identity (2.3) to to rewrite this expression.

$$
\begin{aligned}
\gamma^{[\mu} \eta \wedge \gamma^{\nu]} \eta= & \frac{1}{\operatorname{dim} S} \sum_{\Delta_{k}=-1} \frac{\Delta_{0}\left(\Delta_{0} \Delta_{1}\right)^{k}}{k !} C\left(\gamma^{[\mu} \varphi, \gamma_{(k)} \gamma^{\nu]} \psi\right) \gamma^{(k)} \\
= & \frac{1}{\operatorname{dim} S} \sum_{\Delta_{k}=-1}\left(-\Delta_{0} \Delta_{1}\right)^{k+1}\left(\frac{1}{k !} C\left(\gamma^{\mu \nu(k)} \psi, \varphi\right) \gamma_{(k)}\right. \\
& \left.+\frac{1}{(k-2) !} C\left(\gamma_{(k-2)} \psi, \varphi\right) \gamma^{\mu \nu(k-2)}\right) \\
= & \frac{\left(-\Delta_{0} \Delta_{1}\right)^{n+1}}{\operatorname{dim} S}\left(\frac{1}{(n-2) !} C\left(\gamma^{\mu \nu(n-2)} \eta, \eta\right) \gamma_{(n-2)}+\frac{1}{n !} C\left(\gamma_{(n)} \eta, \eta\right) \gamma^{\mu \nu(n)}\right)
\end{aligned}
$$

The second last equality holds because of (A.1) and the last due to the fact that only the summands with $k=n-2$ and $k=n+2$ survive. Furthermore we needed $1=\Delta_{n}=-\Delta_{n-2}=$ $-\Delta_{n+2}$. Using the duality relation (A.2) to manipulate the first or second summand, we get the following two equivalent expressions

$$
\gamma^{[\mu} \eta \wedge \gamma^{\nu]} \eta=\frac{\left(-\Delta_{0} \Delta_{1}\right)^{n+1}}{n ! \operatorname{dim} S} C\left(\gamma_{(n)} \eta, \eta\right) \gamma^{\mu \nu(n)}\left(\mathbb{1}-(-)^{n} w_{\eta} \gamma^{*}\right)
$$

and

$$
\gamma^{[\mu} \eta \wedge \gamma^{\nu]} \eta=\frac{\left(\Delta_{0} \Delta_{1}\right)^{n+1}}{(n-2) ! \operatorname{dim} S} C\left(\gamma^{\mu \nu(n-2)} \eta, \eta\right) \gamma_{(n-2)}\left(\mathbb{1}-(-)^{n} w_{\eta} \gamma^{*}\right)
$$

where $w_{\eta}$ is defined by $\gamma^{*} \eta=w_{\eta} \eta$. 
Suppose $\operatorname{dim} M=4$. Then (5.1) is self dual if $\eta$ is of negative chirality and anti-self dual if $\eta$ is of positive chirality in the sense that

$$
\frac{1}{2} \epsilon_{\rho \sigma \mu \nu} \gamma^{[\mu} \eta \wedge \gamma^{\nu]} \eta=-w_{\eta} \gamma_{[\rho} \eta \wedge \gamma_{\sigma]} \eta
$$

This yields

Proposition 8. Let $M$ be of dimension four and the pure spinor $\eta$ be of negative (positive) chirality. Then $\mathfrak{B}(R ; \eta, \eta)$ vanishes if the curvature $R$ of $D$ is self dual (resp. anti-self dual).

The last proposition is an extension of the result we obtained in [25] where we examined the four dimensional case with $D=\nabla$ and holonomy $\mathfrak{s u}(2)$ which implies self-duality of the curvature tensor $R^{0}$. Moreover in dimension four there is a further symmetry which yields $\mathfrak{B}\left(R^{0} ; \eta, \eta^{C}\right)=0$ for the parallel pure spinors $\eta$ and its parallel pure charge conjugated $\eta^{C}$.

Self duality of the curvature tensor as a necessary condition for the vanishing of $\mathfrak{B}(R ; \eta, \eta)$ is too restrictive. Suppose $\eta$ is positive so that $\gamma_{[\mu} \eta \wedge \gamma_{\nu]} \eta$ is anti-self dual. This is half of the game. More precisely we find $\gamma_{[\mu} \eta \wedge \gamma_{\nu]} \eta$ in the $\Lambda^{2,0}$ part of anti-self dual forms $\Lambda_{-}^{2} \otimes \mathbb{C}$. Here $\Lambda^{2,0}$ is defined by the complex structure given by $\eta$ (compare [27]). If we use complex matrices $\left\{\gamma^{a}, \gamma^{\bar{a}}\right\}_{1 \leq a, \bar{a} \leq 2}$ associated to this complex structure, i.e. $\gamma^{\bar{a}} \eta=0$, and write $R$ in this frame as $R_{a b}, R_{a \bar{b}}, R_{\bar{a} \bar{b}}$ the necessary condition for the vanishing of $\mathfrak{B}(R ; \eta, \eta)$ is $R_{12}=0$.

If the connection $D$, and so the curvature $R$, is in a real representation the vanishing of the $\Lambda^{2,0}$-part of the curvature is equivalent to two of the three self duality equations. Furthermore we have ${ }^{6} R_{i j}=A R_{\bar{\imath} \bar{j}}^{*} A^{-1}$ and the $\Lambda^{0,2}$ part $R_{\overline{1} \overline{2}}$ vanishes, too. So the condition for the vanishing of $\mathfrak{B}$ reduces to $R \in \Lambda^{1,1}$. The part which prevent the curvature from being self dual is the trace of the $\Lambda^{1,1}$-part. This is due to the isomorphism $\Lambda_{+}^{2}=\Lambda_{0}^{1,1}$, cf. [6].

Similar considerations as in the four dimensional case can be made for arbitrary even dimension. For this we introduce complex coordinates associated to the null space of $\eta,\left\{\gamma^{a}, \gamma^{\bar{a}}\right\}_{1 \leq a, \bar{a} \leq n}$ with $\gamma^{\bar{a}} \eta=0$. The only surviving part of the form which is associated to $\eta$ via the Fierz identity is $C\left(\gamma^{1 \ldots n} \eta, \eta\right) \gamma_{1 \ldots n}$ with only unbarred indices. So (5.1) reads as

$$
\gamma^{\mu} \eta \wedge \gamma^{\nu} \eta \otimes R_{\mu \nu}=\eta^{(n)} \varepsilon^{a_{1} \ldots a_{n}} \gamma_{a_{1} \ldots a_{n-2}}\left(\mathbb{1}-(-)^{n} w_{\eta} \gamma^{*}\right) \otimes R_{a_{n-1} a_{n}}
$$

with $\eta^{(n)}:=\frac{\left(-\Delta_{0} \Delta_{1}\right)^{n+1}}{(n-2) ! \operatorname{dim} S} C\left(\gamma^{1 \ldots n} \eta, \eta\right)$ and $\varepsilon^{a_{1} \ldots a_{n}}$ the totally skew-symmetric symbol of unbarred indices. This yields

Proposition 9. Let $\eta$ be a pure spinor on the even dimensional manifold $M$. Then $\mathfrak{B}(R ; \eta, \eta)$ vanishes if and only if

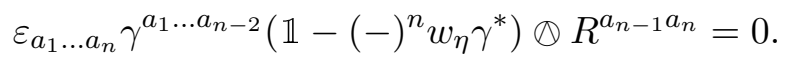

Here the sum is over the unbarred indices with respect to the complex structure given by the pure spinor $\eta$.

A class of connections for which the above is applicable is given in the following corollary. The proof needs the decomposition of $\Lambda^{2}$ which can be taken from the discussion of the four dimensional case.

Corollary 5. Let $D$ be a metric connection on $M$, and suppose it is of holonomy $\mathfrak{s u}(n)$. Then condition (5.2) holds for the two parallel pure spinors.

\footnotetext{
${ }^{6} A$ denotes the matrix which defines the charge conjugation $\varphi^{C}:=A \varphi^{*}$ compare [25].
} 
Using the complex coordinates which have been introduced above, the condition $R_{a b}=0$ as a necessary condition for $\mathfrak{B}(R ; \eta, \eta)=0$ could be seen directly from (4.6). Nevertheless, we used the Fierz identity here to draw a connection to the forms defined by the spinor $\eta$ and to make the condition more precise.

We turn to the torsion dependent term $\mathfrak{D}(\mathcal{T} ; \eta, \eta)$ and distinguish the two cases $\mathcal{T}_{\mu \nu}=$ $\frac{1}{(\ell-2) !} F_{\mu \nu(\ell-2)} \gamma^{(\ell-2)}$ and $\mathcal{T}_{\mu \nu}=\frac{1}{\ell !} F^{(\ell)} \gamma_{\mu \nu(\ell)}$. In both cases we use the Fierz identity as well as (A.1) and condition $\left(2^{\prime}\right)$ above and get after some careful calculations

$$
\begin{aligned}
& \frac{1}{(\ell-2) !} \gamma^{\mu} \eta \wedge F_{\mu \nu(\ell-2)} \gamma^{(\ell-2)} \eta \\
& =\frac{1}{\operatorname{dim} S} \sum_{\Delta_{k}=-1}\left(\Delta_{0} \Delta_{1}\right)^{k+1} \frac{(-)^{\frac{m(m-2 k-1)}{2}}(-)^{k-1}(n-k)}{m !(k-m) !(\ell-m-1) !} \times \\
& \quad \times F_{\nu(m)}^{(\ell-1-m)} C\left(\gamma_{(k-m)(\ell-1-m)} \eta, \eta\right) \gamma^{(m)(k-m)}
\end{aligned}
$$

and

$$
\begin{aligned}
\frac{1}{\ell !} \gamma^{\mu} \eta & \wedge F^{(\ell)} \gamma_{\mu \nu(\ell)} \eta=\frac{1}{\operatorname{dim} S} \sum_{\Delta_{k}=-1}\left(\Delta_{0} \Delta_{1}\right)^{k+1} \frac{(-)^{\frac{m(m-2 k-1)}{2}}(-)^{m+1}(n-k)}{(m+1) !(k-m-1) !(\ell-m) !} \times \\
& \times\left((m+1) F^{(m)}{ }_{(\ell-m)} C\left(\gamma^{(k-m-1)(\ell-m)} \eta, \eta\right) \gamma_{\nu(m)(k-m-1)}\right. \\
& \left.+(-)^{\ell}(\ell-m) F_{(m+1)}{ }^{(\ell-m-1)} C\left(\gamma_{(k-m-1)(\ell-m-1) \nu} \eta, \eta\right) \gamma^{(m+1)(k-m-1)}\right)
\end{aligned}
$$

with $m=\frac{1}{2}(k+\ell-n-1)$. This may be used to get conditions on the forms and their contribution to the connection $D$ to let $\mathfrak{D}(\mathcal{T} ; \eta, \eta)$ vanish. We will not explicitly use this formulas in the next example, but we will see that this would have been possible.

Example 8. We turn again to the case of dimension four. In the case $\ell=3$, i.e. the case of metric connection $D_{\mu}-\nabla_{\mu}^{0}=D_{\mu}^{C}-\nabla_{\mu}^{0}=\mathcal{A}_{\mu}=T_{\mu \nu \kappa} \gamma^{\nu \kappa}$ the term

$$
\gamma^{\mu} \eta \wedge \mathcal{T}_{\mu \nu} \eta=T_{\mu \nu \kappa} \gamma^{\mu} \eta \wedge \gamma^{\kappa} \eta
$$

vanishes in the case of self duality. We recall the decomposition $\Lambda^{2} \otimes \Lambda^{1}=\Lambda^{1} \oplus \Lambda^{3} \oplus \Lambda^{(2,1)}$. If we denote the projections on $\Lambda^{1} \simeq \Lambda^{3}$ and $\Lambda^{3}$ by $\pi_{1}$ and $\pi_{3}$ respectively, we have

$$
T \in \Lambda_{ \pm}^{2} \otimes \Lambda^{1} \Longleftrightarrow * \pi_{1}(T)= \pm \pi_{3}(T) .
$$

This example fits into the discussion of admissible connections, in particular, when we added "non-allowed" forms to the connection in the artificial way (4.1). Moreover if we would have taken an arbitrary one-form $V^{\kappa} \gamma_{\mu \kappa} \gamma^{*}$ and three-form $T_{\mu \nu \kappa} \gamma^{\nu \kappa}$ as contributions to $\mathcal{A}=D-\nabla$, equations (5.3) and (5.4) would have yield exactly the right hand side of (5.5).

As before we may generalize the result to dimensions greater than four. When we consider three-form potentials we see that the $\mathfrak{D}$ - and the $\mathfrak{B}$-term have similar shape. So we get

Proposition 10. Let $\eta$ be a pure spinor and $D$ be constructed by a 3 -form. Then $\mathfrak{D}(\mathcal{T} ; \eta, \eta)=0$ if

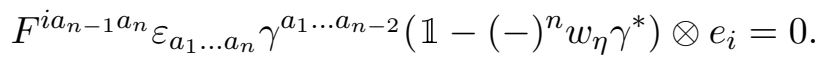

Here the sum over the $a_{*}$ is over the unbarred indices with respect to the complex structure given by the pure spinor $\eta$, and the sum over $i$ is over the complete set of indices. 


\section{Remark 9.}

- (5.6) is solved by $F \in(\mathbf{n} \oplus \overline{\mathbf{n}}) \otimes\left(\mathfrak{s u}(n) \oplus \Lambda^{0,2}\right)$. Of course, the strongly torsion free Example 6 fits into this discussion.

- Propositions 9 and 10 give the conditions on the connection such that the parallel pure spinor yields a differential.

We will make a short comment on the twisted case. Consider a doubled spinor bundle. Suppose there are two pure spinors $\xi, \hat{\xi} \in \Gamma S$, and let $\Xi=(\xi, \hat{\xi})$ be one parallel spinor of the twisted bundle. Furthermore, suppose that the two null-spaces defined by $\xi$ and $\hat{\xi}$ intersect transversally ${ }^{7}$. The necessary condition for $\mathfrak{B}(R ; \Xi, \Xi)$ to vanish is $R=0$. Now suppose that the null spaces of the two spinors have non empty intersection $N$ and the tangent space splits orthogonally into $T=N \oplus N^{\perp}$, i.e. $\Lambda^{2} T=\Lambda^{2} N \oplus N \otimes N^{\perp} \oplus \Lambda^{2} N^{\perp}$. Then the necessary condition reduces and only the part of curvature which acts on $\Lambda^{2} N^{\perp}$ has to vanish.

\subsection{Torsion freeness from brane metrics}

We consider a Lorentzian manifold $M=\left(\mathbb{R}^{D}, g\right)$ such that the coordinates are orthogonal with respect to the metric $g$. Furthermore we consider a spinor connection $D^{C}$ which is determined by a single $q$-form $F$. This $q$-form is Hodge-dual to a vector field $X$, where the Hodge-duality is with respect to only one part of the whole space. Furthermore the metric $g$ shall depend on this vector field in such way that the Christoffel symbols obey $\Gamma_{A B C} \propto X_{A} g_{B C}$. We take $X$ to be the gradient of a function $f$ and use the following ansatz for the metric on $\mathbb{R}^{D}$ :

$$
g=f_{\mu}^{2}(x, y)\left(d x^{\mu}\right)^{2}+f_{i}^{2}(x, y)\left(d y^{i}\right)^{2},
$$

where $\left(x_{\mu}, y_{m}\right)_{0 \leq \mu \leq p, 1 \leq m \leq d}$ is a partition of coordinates into a $(p+1)$-dimensional space-time determined by $\left(x_{\mu}\right)$ and a $d$-dimensional space determined by $\left(y_{m}\right)_{1 \leq m \leq d}$

We discuss two choices for the $q$-form $F$. Either $q=p+2$ with

$$
F_{\mu_{1} \ldots \mu_{p+1} m}=\epsilon_{\mu_{1} \ldots \mu_{p+1}} \partial_{m} f(y)
$$

or $q=d-1$ with

$$
F_{m_{1} \ldots m_{d-1}}=\epsilon_{m_{1} \ldots m_{d-1} m} \delta^{m n} \partial_{n} f(y),
$$

where the function $f$ depends on $\left\{y_{m}\right\}$ only. We call $F$ electric or magnetic field strength in the first or second case, respectively. This notation is due to the fact that the two forms are connected via $F^{(p+2)} \propto *_{D} F^{(d-1)}$. Which values for $p$ are possible to yield an admissible connection in one of the two cases may be checked using Theorem 2 and its extension Theorem 3.

Remark 10. This metric together with the $q$-form for low dimensions is considered in the discussion of $p$-brane solutions of supergravity. E.g. in dimension $D=11$ we have a 5 -brane with magnetic four-form or a 2-brane with electric four-form. More general p-branes may be obtained by using a non-flat metric in the space-time part (pp-waves or AdS) or in the space part (see for example [5] or [12] and references therein).

\footnotetext{
${ }^{7}$ This is true for the parallel pure spinor and its charge conjugated counterpart in the case of Levi-Civita connection of holonomy $\mathfrak{s u}(n)$. In this case $\Xi=\left(\xi, \xi^{C}\right)$ is real and $\mathfrak{B}(R ; \Xi, \Xi)$ does not vanish. This has been used in [25] to show that the real supersymmetric Killing structure is not finite in the case of quaternionic spin representation where a twist of the spinor bundle is necessary to yield a real structure. Nevertheless, it has been shown that in this case there exist two isomorphic finite sub-structures.
} 
We specialize our discussion to the case where the metric is determined by two functions which depend on $\left\{y^{i}\right\}$ only:

$$
g=f_{1}^{2}(y) d x^{2}+f_{2}^{2}(y) d y^{2} .
$$

We refer to the coordinate frame by unchecked indices and to the orthonormal frame by checked indices. The two frames are connected by $e_{\breve{\mu}}=f_{1}(y)^{-1} \partial_{\mu}, e_{\breve{m}}=f_{2}(y)^{-1} \partial_{m}$ and $e^{\check{\mu}}=f_{1}(y) d x^{\mu}$, $e^{\check{m}}=f_{2}(y) d x^{m}$.

The Levi-Civita connection of (5.9) is determined by the Christoffel symbols $\Gamma_{A B C}=\Gamma_{C B A}=$ $\frac{1}{2}\left(\partial_{A} g_{B C}+\partial_{C} g_{B A}-\partial_{B} g_{A C}\right)$

$$
\begin{aligned}
& \Gamma_{\mu \nu i}=-\Gamma_{\mu i \nu}=\partial_{i}\left(\ln f_{1}\right) g_{\mu \nu}, \\
& \Gamma_{i j k}=\partial_{i}\left(\ln f_{2}\right) g_{j k}+\partial_{k}\left(\ln f_{2}\right) g_{i j}-\partial_{j}\left(\ln f_{2}\right) g_{k i}, \\
& \Gamma_{\mu \kappa \nu}=\Gamma_{\mu i j}=\Gamma_{i \mu j}=0,
\end{aligned}
$$

and given by $\nabla_{A}=\partial_{A}+\frac{1}{4} \Gamma_{A B C} \gamma^{B C}$ with

$$
\begin{aligned}
& \nabla_{\mu}=\partial_{\mu}+\frac{1}{2} \Gamma_{\mu \nu i} \gamma^{\nu i}=\partial_{\mu}+\frac{1}{2} \partial_{i}\left(\ln f_{1}\right) f_{1} f_{2}^{-1} \gamma_{\check{\mu}}^{\check{\imath}}, \\
& \nabla_{i}=\partial_{i}+\frac{1}{4} \Gamma_{i \mu \nu} \gamma^{\mu \nu}+\frac{1}{4} \Gamma_{i j k} \gamma^{j k}=\partial_{i}+\frac{1}{2} \partial_{j}\left(\ln f_{2}\right) \gamma_{\check{\imath}}^{\check{\jmath}} .
\end{aligned}
$$

The additional part $-\mathcal{A}^{C}$ of the spinor connection $D^{C}=\nabla-\mathcal{A}^{C}$ is determined by the $q$-form $F$ and given by a linear combination of $F_{\mu A_{1} \ldots A_{q-1}} \gamma^{A_{1} \ldots A_{q-1}}$ and $F^{A_{1} \ldots A_{q}} \gamma_{\mu A_{1} \ldots A_{q}}$.

The magnetic case. We consider the $(d-1)$-form $F$ cf. (5.8) and calculate

$$
\begin{aligned}
-\mathcal{A}_{\mu}^{C} & =\alpha F_{\mu A_{1} \ldots A_{d-2}} \gamma^{A_{1} \ldots A_{d-2}}+\beta F_{A_{1} \ldots A_{d-1}} \gamma_{\mu}{ }^{A_{1} \ldots A_{d-1}} \\
& =\beta \epsilon_{i_{1} \ldots i_{d-1} j^{j k}}\left(\partial_{k} f\right) \gamma_{\mu} \gamma^{i_{1} \ldots i_{d-1}} \\
& =(-)^{d} \beta(d-1) ! f_{2}^{2}\left(\operatorname{det} g_{d}\right)^{-\frac{1}{2}} g^{j k}\left(\partial_{j} f\right) \gamma_{\mu k} \gamma^{[d]} \\
& =(-)^{d} \beta(d-1) !\left(\partial_{j} f\right) f_{1} f_{2}^{2} f_{2}^{-1} f_{2}^{-d} \gamma_{\breve{\mu}} \gamma^{[d]} \\
& =(-)^{d} \beta(d-1) !\left(\partial_{j} f\right) f_{1} f_{2}^{1-d} \gamma_{\breve{\mu}}^{\breve{\jmath}} \gamma^{[d]}
\end{aligned}
$$

as well as

$$
\begin{aligned}
& -\mathcal{A}_{i}^{C}=\alpha F_{i A_{1} \ldots A_{d-2}} \gamma^{A_{1} \ldots A_{d-2}}+\beta F^{A_{1} \ldots A_{d-1}} \gamma_{i A_{1} \ldots A_{d-1}} \\
& =\alpha \epsilon_{i j_{1} \ldots j_{d-2} k} \delta^{k j}\left(\partial_{j} f\right) \gamma^{j_{1} \ldots j_{d-2}}+\beta \epsilon_{j_{1} \ldots j_{d-1} k} \delta^{j k}\left(\partial_{j} f\right) \gamma_{i}^{j_{1 \ldots j} j_{d-1}} \\
& =(-)^{d-1}\left(\alpha(d-2) ! g^{j k} f_{2}^{2}\left(\operatorname{det} g_{d}\right)^{-\frac{1}{2}}\left(\partial_{j} f\right) \gamma_{i k}\right. \\
& \left.+\beta g^{j k} g_{i i^{\prime}} f_{2}^{2}\left(\operatorname{det} g_{d}\right)^{\frac{1}{2}}\left(\partial_{j} f\right) \epsilon_{j_{1} \ldots j_{d-1} k} \epsilon^{i^{\prime} j_{1} \ldots j_{d-1}}\right) \gamma^{[d]} \\
& =(-)^{d-1}\left(\alpha(d-2) !\left(\partial_{j} f\right) f_{2}^{2}\left(\operatorname{det} g_{d}\right)^{-\frac{1}{2}} \gamma_{i}^{\check{\jmath}}+\beta(d-1) !\left(\partial_{i} f\right) f_{2}^{2}\left(\operatorname{det} g_{d}\right)^{-\frac{1}{2}}\right) \gamma^{[d]} \\
& =(-)^{d-1}\left(\alpha(d-2) !\left(\partial_{j} f\right) f_{2}^{2-d} \gamma_{i}^{\breve{\jmath}}+\beta(d-1) !\left(\partial_{i} f\right) f_{2}^{2-d} \gamma^{[d]}\right) \text {. }
\end{aligned}
$$

From now on we suppose that at least one of the two factors in the brane ansatz is even dimensional. The matrix $\gamma^{[d]}=\frac{1}{d !} \epsilon_{i_{1} \ldots i_{d}} \gamma^{i_{1} \ldots i_{d}}$ is connected to the volume element of the space factor in $M$ and obeys

$$
\gamma^{[d]} \gamma_{j}=(-)^{d+1} \gamma_{j} \gamma^{[d]} \quad \text { and } \quad \gamma^{[d]} \gamma_{\mu}=(-)^{d} \gamma_{\mu} \gamma^{[d]}
$$

We choose $\varepsilon \in\{1, i\}$ such that $\left(\varepsilon \gamma^{[d]}\right)^{2}=\mathbb{1}$. Then $\Pi^{ \pm}=\frac{1}{2}\left(\mathbb{1} \pm \varepsilon \gamma^{[d]}\right)$ is the projection on one half of the spinor bundle. When we denote the spinor bundle of $M$, of its $d$-dimensional factor, and of its $(p+1)$-dimensional factor by $S_{D}, S_{d}$ and $S_{p+1}$, respectively, we have

$$
S_{D}^{ \pm}=\Pi^{ \pm}\left(S_{D}\right)= \begin{cases}S_{d}^{ \pm} \otimes S_{p+1} & \text { if } d \text { even } \\ S_{d} \otimes S_{p+1}^{ \pm} & \text {if } d \text { odd }\end{cases}
$$


Furthermore we suppose that $f_{1}, f_{2}, f$ and $\alpha, \beta$ obey

$$
\begin{aligned}
X_{i} & :=\partial_{i}\left(\ln f_{1}\right) f_{1} f_{2}^{-1}=(-)^{d} \delta_{1} \frac{2}{\varepsilon} \beta(d-1) !\left(\partial_{i} f\right) f_{1} f_{2}^{1-d}, \\
Y_{i} & :=\partial_{i}\left(\ln f_{2}\right)=-(-)^{d} \delta_{2} \frac{2}{\varepsilon} \alpha(d-2) !\left(\partial_{i} f\right) f_{2}^{2-d}
\end{aligned}
$$

for some choice of signs $\delta_{1}, \delta_{2} \in\{ \pm 1\}$.

Remark 11. (5.10) can be obtained by the ansatz $f_{\ell}(y)=e^{\alpha_{\ell} u(y)}$ which yields the following system for the constants $\alpha_{\ell}$ :

$$
\alpha_{1}=(-)^{d} \delta_{1} \frac{2}{\varepsilon} \beta(d-1) ! \alpha_{3}, \quad \alpha_{2}=-(-)^{d} \delta_{2} \frac{2}{\varepsilon} \alpha(d-2) ! \alpha_{3}, \quad \alpha_{3}=(d-2) \alpha_{2} .
$$

For $d=5$ we deal with a four-form $F$ which leads to an admissible connection when we have $\Delta_{0} \Delta_{1}=-1$. Then a possible solution for $\delta_{1}=-\delta_{2}=-1$ and $\varepsilon=i$ is $\beta=-\frac{i}{288}, \alpha=\frac{8 i}{288}$, $\alpha_{1}=-\frac{1}{6}, \alpha_{2}=\frac{1}{3}$, and $\alpha_{3}=1$. In dimension eleven this is the supergravity M5-brane solution.

With (5.10) the connection $D^{C}$ is given by

$$
D_{\mu}^{C}=\partial_{\mu}+X_{i} \gamma_{\breve{\mu}}^{\check{\imath}} \Pi^{ \pm}, \quad D_{i}^{C}=\partial_{i}+Y_{j} \gamma_{i}^{\check{\jmath}} \Pi^{ \pm}+\delta_{2} \varepsilon \frac{(d-1) \beta}{2 \alpha} Y_{i} \gamma^{[d]} .
$$

The signs $\delta_{*}$ in (5.10) determine which projection is present. Nevertheless, the projections should be the same in both terms.

Proposition 11. 1. The holonomy of the connection (5.11) is given by

$$
\mathfrak{h o l}=\mathfrak{s o}(d) \ltimes \begin{cases}(p+1) \cdot 2^{\frac{d-1}{2}} \cdot S_{d} & \text { if } d \text { odd, }(\text { i.e. }(p+1) \text { even }), \\ (p+1) \cdot 2^{\frac{d}{2}-1} \cdot \tilde{S}_{d} & \text { if } d \equiv 0 \bmod 4, \\ (p+1) \cdot 2^{\frac{d}{2}} \cdot \tilde{S}_{d} & \text { if } d \equiv 2 \bmod 4,\end{cases}
$$

Here $\mathfrak{s o}(d) \subset \mathfrak{s l}\left(S_{D}^{ \pm}\right)$and for d even $\tilde{S}_{d}$ denotes the $2^{\frac{d}{2}-1}$-dimensional (not specified) half spinor representation $S_{d}^{ \pm}$.

2. The torsion of the connection $D$ - the charge conjugated of (5.11) - is given by

$$
\begin{aligned}
& \mathcal{T}_{\mu \nu}=\delta_{1} \varepsilon f_{1} f_{2} X \cdot \gamma_{\breve{\mu} \check{\nu}} \gamma^{[d]}, \\
& \mathcal{T}_{\mu i}=-\delta_{1} \varepsilon f_{2} X_{i} \gamma_{\check{\mu}} \gamma^{[d]}=\delta_{2} \varepsilon(d-1) \beta \alpha^{-1} f_{1} Y_{i} \gamma_{\breve{\mu}} \gamma^{[d]}, \\
& \mathcal{T}_{i j}=\delta_{2} \varepsilon f_{2} Y_{k} \gamma_{\check{\iota} \breve{\jmath} \gamma^{[d]}} .
\end{aligned}
$$

Proof. The bracket $\left[D_{\mu}, D_{\nu}\right]$ vanishes due to $\Pi^{ \pm} \gamma_{\breve{\mu} \check{\imath}}=\gamma_{\check{\mu} \check{\imath}} \Pi^{\mp}$ and $\Pi^{\mp} \Pi^{ \pm}=0$ whereas $\left[D_{\mu}, D_{i}\right]$ is given by

$$
\begin{aligned}
& {\left[\partial_{\mu}+X_{j} \gamma_{\breve{\mu}}^{\check{\jmath}} \Pi^{ \pm}, \partial_{i}+Y_{j} \gamma_{\check{\imath}}^{\check{\jmath}} \Pi^{ \pm}+\delta_{2} \varepsilon \frac{(d-1) \beta}{2 \alpha} Y_{i} \gamma^{[d]}\right]} \\
& =\left[X_{j} \gamma_{\breve{\mu}}^{\check{\jmath}} \Pi^{ \pm}, Y_{k} \gamma_{\bar{\imath}}^{\check{k}} \Pi^{ \pm}\right]+\left[X_{j} \gamma_{\breve{\mu}}^{\check{\jmath}} \Pi^{ \pm}, \delta_{2} \varepsilon \frac{(d-1) \beta}{2 \alpha}(d-1) Y_{i} \gamma^{[d]}\right]-\partial_{i} X_{j} \gamma_{\breve{\mu}}{ }^{\breve{ }} \Pi^{ \pm} \\
& =X_{j} Y_{k}\left[\gamma_{\breve{\mu}}^{\check{\jmath}} \Pi^{ \pm}, \gamma_{\check{\imath}}^{\check{k}} \Pi^{ \pm}\right]+\delta_{2} \frac{(d-1) \beta}{2 \alpha} Y_{i} X_{j}\left[\gamma_{\breve{\mu}}^{\check{\jmath}} \Pi^{ \pm}, \varepsilon \gamma^{[d]}\right]-\partial_{i} X_{j} \gamma_{\breve{\mu}}^{\check{\jmath}} \Pi^{ \pm} \\
& =X_{j} Y_{k}\left(\gamma_{\breve{\mu}}^{\breve{\jmath}} \Pi^{ \pm} \gamma_{\bar{\imath}}^{\check{k}} \Pi^{ \pm}-\gamma_{\grave{k}}^{\check{k}} \Pi^{ \pm} \gamma_{\breve{\mu}}^{\breve{\jmath}} \Pi^{ \pm}\right) \\
& +\delta_{2} \frac{(d-1) \beta}{2 \alpha} Y_{i} X_{j}\left(\gamma_{\check{\mu}}^{\check{\jmath}} \Pi^{ \pm} \varepsilon \gamma^{[d]}-\varepsilon \gamma^{[d]} \gamma_{\check{\mu}}^{\check{\jmath}} \Pi^{ \pm}\right)-\partial_{i} X_{j} \gamma_{\check{\mu}}^{\check{\jmath}} \Pi^{ \pm}
\end{aligned}
$$




$$
\begin{aligned}
& =X_{j} Y_{k} \gamma_{\breve{\mu}}^{\breve{\jmath}} \gamma_{\check{i}}^{\check{k}} \Pi^{ \pm} \pm \delta_{2} \frac{(d-1) \beta}{2 \alpha} Y_{i} X_{j} \gamma_{\breve{\mu}}^{\breve{\jmath}} \Pi^{ \pm}-\partial_{i} X_{j} \gamma_{\breve{\mu}}^{\breve{\jmath}} \Pi^{ \pm} \\
& =X_{j} Y_{k} \gamma_{\breve{\mu}}\left(-\delta_{\check{\imath}}^{\check{\jmath}} \gamma^{\check{k}}+g^{\check{j} \check{k}} \gamma_{\check{\imath}}\right) \Pi^{ \pm} \pm \delta_{2} \frac{(d-1) \beta}{2 \alpha} Y_{i} X_{j} \gamma_{\breve{\mu}}^{\breve{\jmath}} \Pi^{ \pm}-\partial_{i} X_{j} \gamma_{\breve{\mu}}^{\check{\jmath}} \Pi^{ \pm} \\
& =-X_{i} Y_{j} \gamma_{\breve{\mu}}^{\check{\jmath}} \Pi^{ \pm}+X_{k} Y^{k} f_{2}^{2} \gamma_{\breve{\mu} \breve{\imath}} \Pi^{ \pm} \pm \delta_{2} \frac{(d-1) \beta}{2 \alpha} Y_{i} X_{j} \gamma_{\breve{\mu}}^{\breve{\jmath}} \Pi^{ \pm}-\partial_{i} X_{j} \gamma_{\breve{\mu}}^{\breve{\jmath}} \Pi^{ \pm} \\
& =\left(\left(\frac{ \pm \delta_{2}(d-1) \beta-2 \alpha}{2 \alpha} X_{i} Y_{j}-\partial_{i} X_{j}\right) \gamma_{\breve{\mu}}^{\check{\jmath}}+X_{k} Y^{k} f_{2}^{2} \gamma_{\breve{\mu} \check{\imath}}\right) \Pi^{ \pm} \text {. }
\end{aligned}
$$

Here we used $\gamma_{\breve{\mu} \check{\imath}} \gamma^{[d]}=-\gamma^{[d]} \gamma_{\breve{\mu} \check{\imath}}$ and $X_{j} Y_{k}=X_{k} Y_{j}$. When we calculate $\left[D_{\mu}, D_{i}\right]$ we furthermore use $\gamma_{\imath \check{\jmath}} \gamma^{[d]}=\gamma^{[d]} \gamma_{\check{\imath} \jmath}$. This yields

$$
\begin{aligned}
& {\left[\partial_{i}+Y_{k} \gamma_{\check{i}}^{\check{k}} \Pi^{ \pm}+\delta_{2} \varepsilon \frac{(d-1) \beta}{2 \alpha} Y_{i} \gamma^{[d]}, \partial_{j}+Y_{\ell} \gamma_{\check{\jmath}}^{\check{\ell}} \Pi^{ \pm}+\delta_{2} \varepsilon \frac{(d-1) \beta}{2 \alpha} Y_{j} \gamma^{[d]}\right]} \\
& =\left(\partial_{i} Y_{\ell}\right) \gamma_{\check{\jmath}}^{\check{\ell}} \Pi^{ \pm}-\left(\partial_{j} Y_{\ell}\right) \gamma_{\grave{\ell}}^{\check{\ell}} \Pi^{ \pm}+\delta_{2} \varepsilon \frac{(d-1) \beta}{2 \alpha}\left(\partial_{i} Y_{j}-\partial_{j} Y_{i}\right) \gamma^{[d]}+Y_{k} Y_{\ell}\left[\gamma_{\check{i}}^{\check{k}}, \gamma_{\check{\jmath}}^{\check{\ell}}\right] \Pi^{ \pm}
\end{aligned}
$$

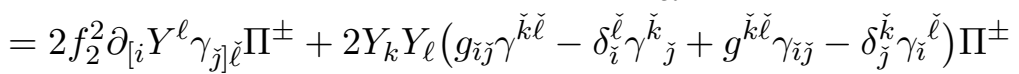

$$
\begin{aligned}
& =2\left(f_{2}^{2} \partial_{[i} Y^{k} \gamma_{\check{\jmath}] \check{k}}+f_{2}^{-2} Y_{k} Y^{k} \gamma_{\check{\imath} \check{\jmath}}+2 Y_{k} Y_{[i} \gamma_{\check{\jmath}]}^{\check{k}}\right) \Pi^{ \pm} \text {. }
\end{aligned}
$$

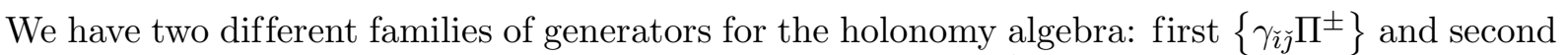
$\left\{\gamma_{\breve{\mu} \breve{j}} \Pi^{ \pm}\right\}$. The first one generates a $\mathfrak{s o}(d)$ sub algebra of $\mathfrak{s l}\left(S_{D}^{ \pm}\right) \subset \mathfrak{s l}\left(S_{D}\right)$.

Suppose $d$ is odd. The action of $\mathfrak{s o}(d)$ on the second family generates the commuting set

$$
\operatorname{span}\left\{\gamma_{\check{\mu} \check{\iota}_{1} \ldots \check{\nu}_{r}} \Pi^{ \pm} \mid r \text { odd }\right\} \simeq C \ell_{d}^{\text {odd }}
$$

The action of $\mathfrak{s o}(d)$ on this set is given by right multiplication

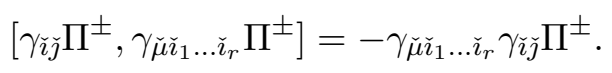

As a spin module via right (or left) multiplication the Clifford algebra is isomorphic to a direct sum of copies of the minimal spinor representation $S_{d}$ and so is the $2^{d-1}$-dimensional odd part due to Spin-invariance.

The minimal representation $S_{d}$ is of dimension $2^{\frac{d-1}{2}}$. Therefore, the commuting set is isomorphic to $(p+1) \cdot 2^{\frac{d-1}{2}} S_{d}$ as representation space.

Suppose $d$ is even. Consider once more the set generated by the action of $\mathfrak{s o}(d)$ on the second family. If $\left(\gamma^{[d]}\right)^{2}=\mathbb{1}(d \equiv 0 \bmod 4)$ we have $\gamma^{\text {odd }} \Pi^{ \pm} \propto \gamma^{\text {odd }} \gamma^{[d]} \Pi^{ \pm}=\gamma^{\text {odd }} \Pi^{ \pm}$in the other case $(d \equiv 2 \bmod 4)$ there is an extra $i$-factor in the proportionality. Therefore, the commuting set is (5.13) of dimension $2^{d-1}$ if $d \equiv 2 \bmod 4$, and only one half of this if $d \equiv 0 \bmod 4$ due to the duality above.

The minimal representation $S_{d}^{ \pm}$is of dimension $2^{\frac{d}{2}-1}$. Therefore, as representation space the commuting set is isomorphic to $(p+1) \cdot 2^{\frac{d}{2}-1} \tilde{S}_{d}$ if $d \equiv 0 \bmod 4$ and to $(p+1) \cdot 2^{\frac{d}{2}} \tilde{S}_{d}$ if $d \equiv 2 \bmod 4$.

The torsion of the admissible connection $D=\nabla+\mathcal{A}$ is given by $\mathcal{T}_{A B}=a d_{\mathcal{A}_{A}}^{C} \gamma_{B}=\mathcal{A}_{A} \gamma_{B}+$ $\gamma_{B} \mathcal{A}_{A}^{C}$. We have

$$
-\mathcal{A}_{\mu}^{C}=\frac{\delta_{1} \varepsilon}{2} X_{i} \gamma_{\check{\mu}}^{\check{l}} \gamma^{[d]}, \quad-\mathcal{A}_{i}^{C}=\frac{\delta_{2} \varepsilon}{2} Y_{j} \gamma_{\grave{r}}^{\check{\jmath}} \gamma^{[d]}+\frac{\delta_{2} \varepsilon(d-1) \beta}{2 \alpha} Y_{i} \gamma^{[d]} .
$$

Due to Theorem 2 we have $\Delta_{d-1} \Delta_{1}=-1$ or equivalently $\Delta_{d} \Delta_{0}=(-)^{d}$ which yields

$$
-\mathcal{A}_{\mu}=\frac{\delta_{1} \varepsilon}{2} X_{i}\left(\gamma_{\check{\mu}}^{\check{\imath}} \gamma^{[d]}\right)^{C}=(-)^{d} \frac{\delta_{1} \varepsilon}{2} X_{i} \gamma_{\check{\mu}}^{\check{\imath}} \gamma^{[d]},
$$




$$
\begin{aligned}
-\mathcal{A}_{i} & =\frac{\delta_{2} \varepsilon}{2} Y_{j}\left(\gamma_{i}^{\check{\jmath}} \gamma^{[d]}\right)^{C}+\frac{\delta_{2} \varepsilon(d-1) \beta}{2 \alpha} Y_{i}\left(\gamma^{[d]}\right)^{C} \\
& =-(-)^{d} \frac{\delta_{2} \varepsilon}{2} Y_{j} \gamma_{i}^{\check{\jmath}} \gamma^{[d]}+(-)^{d} \frac{\delta_{2} \varepsilon(d-1) \beta}{2 \alpha} Y_{i} \gamma^{[d]} .
\end{aligned}
$$

This is used to calculate the torsion of the brane connection:

$$
\begin{aligned}
\mathcal{T}_{\mu \nu} & =\mathcal{A}_{\mu} \gamma_{\nu}+\gamma_{\nu} \mathcal{A}_{\mu}^{C}=-\frac{\delta_{1} \varepsilon}{2} X_{i}\left((-)^{d} \gamma_{\check{\mu}}^{\check{L}} \gamma^{[d]} \gamma_{\nu}+\gamma_{\nu} \gamma_{\check{\mu}}^{\check{L}} \gamma^{[d]}\right) \\
& =-\frac{\delta_{1} \varepsilon}{2} f_{1} X_{i}\left(-\gamma_{\check{\mu}} \gamma_{\check{\nu}} \gamma^{\check{\nu}} \gamma^{[d]}+\gamma_{\check{\nu}} \gamma_{\check{\mu}} \gamma^{\check{\nu}} \gamma^{[d]}\right)=\delta_{1} \varepsilon f_{1} f_{2} X \cdot \gamma_{\breve{\mu} \check{\nu}} \gamma^{[d]}
\end{aligned}
$$

as well as

$$
\begin{aligned}
\mathcal{T}_{\mu i} & =\mathcal{A}_{\mu} \gamma_{i}+\gamma_{i} \mathcal{A}_{\mu}^{C}=-\frac{\delta_{1} \varepsilon}{2} X_{j}\left((-)^{d} \gamma_{\breve{\mu}}{ }^{\breve{\jmath}} \gamma^{[d]} \gamma_{i}+\gamma_{i} \gamma_{\breve{\mu}}^{\check{\jmath}} \gamma^{[d]}\right) \\
& =\frac{\delta_{1} \varepsilon}{2} f_{2} X_{j}\left(\gamma^{\check{\jmath}} \gamma_{\check{\imath}}+\gamma_{\check{\imath}} \gamma^{\breve{\jmath}}\right) \gamma_{\breve{\mu}} \gamma^{[d]}=-\delta_{1} \varepsilon f_{2} X_{i} \gamma_{\breve{\mu}} \gamma^{[d]} .
\end{aligned}
$$

Last but not least we have

$$
\begin{aligned}
& \mathcal{T}_{i j}=\mathcal{A}_{i} \gamma_{j}+\gamma_{j} \mathcal{A}_{i}^{C} \\
& =\frac{\delta_{2} \varepsilon}{2} Y_{k}\left((-)^{d} \gamma_{\grave{i}}^{\check{k}} \gamma^{[d]} \gamma_{j}-\gamma_{j} \gamma_{\grave{i}}^{\check{k}} \gamma^{[d]}\right)-\frac{\delta_{2} \varepsilon(d-1) \beta}{2 \alpha} Y_{i}\left((-)^{d} \gamma^{[d]} \gamma_{j}+\gamma_{j} \gamma^{[d]}\right) \\
& =-\frac{\delta_{2} \varepsilon}{2} f_{2} Y_{k}\left(\gamma_{\grave{\imath}}^{\breve{k}} \gamma_{\check{\jmath}}+\gamma_{\check{\jmath}} \gamma_{\check{\imath}}^{\breve{k}}\right) \gamma^{[d]}=\delta_{2} \varepsilon f_{2} Y_{k} \gamma_{\check{\check{\jmath}}}^{\check{\jmath}} \gamma^{[d]} \text {. }
\end{aligned}
$$

Corollary 6. The spinors which are parallel with respect to the connection (5.11) form a subspace of the kernel of $\Pi^{ \pm}$. Explicitly we have $\eta(y)=f(y) \eta_{0}$ with constant $\eta_{0} \in S_{D}^{\mp}$ and $f$ obeys $\partial_{i} f= \pm \delta_{2} \frac{(d-1) \beta}{2 \alpha} Y_{i} f$.

The electric case. Due to the fact that the electric $(p+2)$-form is dual to the magnetic $(d-1)$-form we will only give a rough sketch of what is used to get a similar result. We will assume that at least one of the factors is even dimensional. Then we have the duality relation induced by $\gamma^{[d]} \gamma^{[D]} \propto \gamma^{[p+1]}$. For a suitable choice of $X$ and $Y$ we get

$$
D_{\mu}^{C}=\partial_{\mu}+X_{i} \gamma_{\check{\mu}}^{\check{\imath}} \hat{\Pi}^{ \pm}, \quad D_{i}^{C}=\partial_{\mu}+Y_{j} \gamma_{\breve{\mu}}^{\check{\jmath}} \hat{\Pi}^{ \pm}+\alpha Y_{i} \gamma^{[p+1]}
$$

which is of the same type as in the magnetic case. The projections are given by

$$
\hat{\Pi}^{ \pm}: S_{D}=S_{d} \otimes S_{p+1} \rightarrow S_{D}^{ \pm}= \begin{cases}S_{d} \otimes S_{p+1}^{ \pm} & \text {if }(p+1) \text { is even } \\ S_{d}^{ \pm} \otimes S_{p+1} & \text { if }(p+1) \text { is odd }\end{cases}
$$

The expressions for the holonomy and the torsion can be taken directly from Proposition 11.

In the remaining part of this section we analyze in what way we have to restrict the set of parallel spinors to yield a torsion free subset $\mathcal{K}$ in the sense of Definition 5. I.e. we look for solutions of $\mathfrak{D}(\mathcal{T}, \eta, \xi)=0$ or equivalently

$$
\begin{aligned}
& \mathcal{T}_{i \mu} \eta \wedge \gamma^{\mu} \xi+\mathcal{T}_{i j} \eta \wedge \gamma^{j} \xi+(\eta \leftrightarrow \xi)=0, \\
& \mathcal{T}_{\mu i} \eta \wedge \gamma^{i} \xi+\mathcal{T}_{\mu \nu} \eta \wedge \gamma^{\nu} \xi+(\eta \leftrightarrow \xi)=0
\end{aligned}
$$

with $\mathcal{T}_{A B}$ given by (5.12). We discuss the four summands separately and get:

$$
\mathcal{T}_{i \mu} \eta \wedge \gamma^{\mu} \xi=-\delta_{1} \varepsilon f_{2} X_{i} \gamma_{\check{\mu}} \gamma^{[d]} \eta \wedge \gamma^{\mu} \xi=\delta_{1} \varepsilon f_{2} f_{1}^{-1} X_{i} \gamma_{\check{\mu}} \xi \wedge \gamma^{\check{\mu}} \gamma^{[d]} \eta
$$




$$
=\delta_{1} \varepsilon f_{2} X_{i} \gamma_{\breve{\mu}} \gamma^{[d]} \xi \wedge \gamma^{\mu} \eta=-\mathcal{T}_{i \mu} \xi \wedge \gamma^{\mu} \eta
$$

where the second last equality holds because the spinors are of the same chirality with respect to $\gamma^{[d]}$.

From this result we see that the first summand in (5.14) vanishes after symmetrization over the spinorial entries. With $Z_{k}=\delta_{2} \varepsilon f_{2} Y_{k}$ we keep on calculating

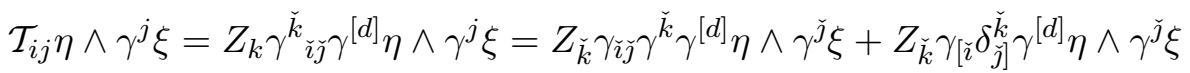

$$
\begin{aligned}
& =\gamma_{\check{\imath} \check{\jmath}} Z \cdot \gamma^{[d]} \eta \wedge \gamma^{\check{\jmath}} \xi+\gamma_{\check{\imath}} \gamma^{[d]} \eta \wedge Z \xi-Z_{\check{\imath}} \gamma_{\check{\jmath}} \gamma^{[d]} \eta \wedge \gamma^{\check{\jmath}} \xi \text {. }
\end{aligned}
$$

The last summand vanishes when we symmetrize with respect to $\eta$ and $\xi$. Furthermore we have

$$
T_{\mu i} \eta \wedge \gamma^{i} \xi=-\delta_{1} \varepsilon f_{2} \gamma_{\check{\mu}} \gamma^{[d]} \eta \wedge(X \xi)=-(-)^{d} \delta_{1} \varepsilon f_{2} \gamma^{[d]} \gamma_{\check{\mu}} \eta \wedge(X \xi)
$$

and

$$
T_{\mu \nu} \eta \wedge \gamma^{\nu} \xi=\delta_{1} \varepsilon f_{2} f_{1} X \gamma_{\check{\mu} \check{\nu}} \gamma^{[d]} \eta \wedge \gamma^{\nu} \xi=(-)^{d+1} \delta_{1} \varepsilon f_{2} \gamma_{\check{\mu} \check{\nu}} \gamma^{[d]}(X \eta) \wedge \gamma^{\check{\nu}} \xi
$$

If we put all this together and use $X \propto Z$ then equations (5.14) reduce to

$$
\begin{aligned}
& \gamma_{\check{\imath} \check{\jmath}} \gamma^{[d]}(X \eta) \wedge \gamma^{\check{\jmath}} \xi+\gamma^{[d]} \gamma_{\check{\imath}} \eta \wedge(X \xi)+(\eta \leftrightarrow \xi)=0, \\
& \gamma_{\check{\mu} \check{\nu}} \gamma^{[d]}(X \eta) \wedge \gamma^{\check{\nu}} \xi+\gamma^{[d]} \gamma_{\check{\mu}} \eta \wedge(X \xi)+(\eta \leftrightarrow \xi)=0 .
\end{aligned}
$$

We collect the brane-example in the following theorem.

Theorem 4. Consider the manifold $M$ which is $\mathbb{R}^{p+1} \times \mathbb{R}^{d}$ equipped with the p-brane metric (5.7) and denote its spinor bundle by $S$. Let $F$ be a magnetic $(d-1)$-form on $M$, i.e. it is $*_{d}$-dual to a gradient field $X(y)$ on the transversal space $\mathbb{R}^{d}$. The form $F$ and the metric are compatible such that they define an admissible connection $D$ on the spinor bundle cf. (5.11). Then the space $\mathcal{K}$ given by

$$
\mathcal{K}=\left\{\eta \in \Gamma S \mid D^{C} \eta=0, X \eta=0\right\}
$$

is admissible and torsion free.

\section{Outlook}

As stated in the introduction admissible spinorial connections, i.e. connections with further symmetry condition on its torsion c.f. Definition 3, are basic objects when we look for infinitesimal automorphisms of the underlying manifold constructed from parallel spinors, compare Theorem 1. This condition may be relaxed by considering admissible pairs cf. Definition 4 . The notion of torsion enters naturally, when we look at commutators of vector fields on supermanifolds constructed from the spinor bundle. This will be one tool in constructing a purely geometric representation of the supersymmetry algebra extending the work of [2] or [25]. Work on this construction is in progress.

\section{A Useful identities and symmetries for Clifford multiplication and charge conjugation}

In this appendix we collect some identities concerning gamma matrices as well as some properties of the symmetry of the morphisms $(2.1)^{8}$.

\footnotetext{
${ }^{8}$ We note that most of the formulas are valid without additional (det $g$ )-factors only if the indices belong to an orthonormal frame (compare the calculations in Section 5.3).
} 
For the Clifford multiplication we use the convention $\gamma_{\{\mu} \gamma_{\nu\}}=-g_{\mu \nu}$ which yields

$$
\left.\gamma_{\mu_{1} \ldots \mu_{k}} \gamma^{\nu_{1} \ldots \nu_{\ell}}=\sum_{m=0}^{\min \{k, \ell\}} \frac{(-)^{\frac{m(m-2 k-1)}{2}} k ! \ell !}{m !(k-m) !(\ell-m) !} \delta_{\left[\mu_{1} \ldots \mu_{m}\right.}^{\left[\nu_{1} \ldots \nu_{m}\right.} \gamma_{\left.\mu_{m+1} \ldots \mu_{k}\right]} \nu_{m+1} \ldots \nu_{\ell}\right]
$$

We have

$$
\gamma_{\mu_{1} \ldots \mu_{k}}=\frac{1}{(D-k) !}(-)^{\frac{k(k+1)}{2}}(-)^{\frac{D(D+1)}{2}} \epsilon_{\mu_{1} \ldots \mu_{D}} \gamma^{\mu_{k+1} \ldots \mu_{D}} \gamma^{[D]}
$$

with

$$
\gamma^{[D]}:=\gamma^{1} \cdots \gamma^{D}=\frac{1}{D !} \epsilon_{\mu_{1} \ldots \mu_{D}} \gamma^{\mu_{1}} \cdots \gamma^{\mu_{D}}
$$

This matrix obeys $\left(\gamma^{[D]}\right)^{2}=(-)^{\frac{D(D+1)}{2}}+t$ where $t$ denotes the amount of time-like directions in the metric. For $D$ odd $\gamma^{[D]}$ is proportional to $\mathbb{1}$. For $D=2 n$ even we define the modified matrix

$$
\gamma^{*}= \begin{cases}\gamma^{[2 n]} & \tilde{\sigma} \equiv 0 \bmod 4 \\ i \gamma^{[2 n]} & \tilde{\sigma} \equiv 2 \bmod 4\end{cases}
$$

where $\tilde{\sigma}$ denotes the signature of the metric $g$. It obeys

$$
\gamma^{*} \gamma^{(k)}=(-)^{k} \gamma^{(k)} \gamma^{*} \quad \text { and } \quad\left(\gamma^{*}\right)^{2}=\mathbb{1}
$$

and yields a splitting of the spinors in the two eigenspaces $S=S^{+} \oplus S^{-}$.

The symmetry property (2.2) implies

$$
\begin{aligned}
& \Delta_{k}=-1 \Leftrightarrow k \in\left\{4 m-\Delta_{1}, 4 m+1+\Delta_{0}\right\}, \\
& \Delta_{0} \Delta_{k}=-1 \Leftrightarrow k \in\left\{4 m+2,4 m-\Delta_{0} \Delta_{1}\right\}, \\
& \Delta_{1} \Delta_{k}=-1 \Leftrightarrow \Delta_{0} \Delta_{k-1}=(-)^{k} \Leftrightarrow k \in\left\{4 m+3,4 m+1+\Delta_{0} \Delta_{1}\right\} .
\end{aligned}
$$

The symmetries $\Delta_{k}$ and $\Delta_{D-k}$ are connected via

$$
\Delta_{D-k}=(-)^{\frac{D(D-1)}{2}}(-)^{D k}(-)^{k}\left(\Delta_{0} \Delta_{1}\right)^{D} \Delta_{k} .
$$

This yields

$$
\begin{array}{ll}
\Delta_{k}=(-)^{n+k} \Delta_{D-k}=\Delta\left(\gamma^{(D-k)} \gamma^{*}\right) & \text { if } D=2 n \text { even, } \\
\Delta_{k}=\Delta_{D-k} & \text { if } D=2 n+1 \text { odd. }
\end{array}
$$

Introducing the complex coordinates $\gamma^{a}=\gamma^{a}+i \gamma^{a+n}$ and $\gamma^{\bar{a}}=\gamma^{a}-i \gamma^{a+n}$, for $a, \bar{a}=1, \ldots, n$, yields

$$
\begin{aligned}
& \gamma^{\{a} \gamma^{b\}}=\gamma^{\{\bar{a}} \gamma^{\bar{b}\}}=0, \quad \gamma^{\{a} \gamma^{\bar{b}\}}=-2 g^{a \bar{b}} \\
& (-)^{\frac{\sigma(\sigma-1)}{2}} \gamma^{*}=\gamma^{1 \overline{1}} \cdots \gamma^{n \bar{n}}=\left(\mathbb{1}+\gamma^{1} \gamma^{\overline{1}}\right) \cdots\left(\mathbb{1}+\gamma^{n} \gamma^{\bar{n}}\right) \\
& \gamma^{1 \ldots n} \gamma^{*}=\gamma^{1 \ldots n}, \quad \gamma^{\overline{1} \ldots \bar{n}} \gamma^{*}=(-)^{n} \gamma^{\overline{1} \ldots . \bar{n}} .
\end{aligned}
$$

We use the following modified Pauli-matrices if we are forced to modify the charge conjugation to change symmetries:

$$
\tau_{0}=\sigma_{0}=\left(\begin{array}{ll}
1 & \\
& 1
\end{array}\right), \quad \tau_{1}=\sigma_{1}=\left(\begin{array}{cc} 
& 1 \\
1 &
\end{array}\right),
$$




$$
\tau_{2}=i \sigma_{2}=\left(\begin{array}{cc} 
& 1 \\
-1 &
\end{array}\right), \quad \tau_{3}=\sigma_{3}=\left(\begin{array}{ll}
1 & \\
& -1
\end{array}\right)
$$

To these matrices we associate two kind of signs. The first sign is $\varepsilon_{i k}$ which we get by permuting two of the matrices, i.e. $\tau_{i} \tau_{k}=\varepsilon_{i k} \tau_{k} \tau_{i}$, and the second is $\varepsilon_{k}$ which indicates the symmetry of $\tau_{k}$

$$
\varepsilon_{i k}=\left(\begin{array}{cccc}
1 & 1 & 1 & 1 \\
1 & 1 & -1 & -1 \\
1 & -1 & 1 & -1 \\
1 & -1 & -1 & 1
\end{array}\right), \quad \varepsilon_{k}=\left(\begin{array}{c}
1 \\
1 \\
-1 \\
1
\end{array}\right)
$$

\section{Acknowledgements}

The author would like to thank Vicente Cortés and Mario Listing for useful discussions on the topic of this text.

[1] Alekseevsky D.V., Cortés V., Devchand C., van Proeyen A., Polyvector super-Poincaré algebras, Comm. Math. Phys., 2005, V.253, 385-422, hep-th/0311107.

[2] Alekseevsky D.V., Cortés V., Devchand C., Semmelmann U., Killing spinors are Killing vector fields in Riemannian supergeometry, J. Geom. Phys., 1998, V.26, 37-50, dg-ga/9704002.

[3] Alekseevsky D.V., Cortés V., Classification of $N$-extended Poincaré algebras and bilinear invariants of the spinor representation of Spin $(p, q)$, Comm. Math. Phys., 1997, V.183, 477-510, math.RT/9511215.

[4] Alishahiha M., Mohammad A. Ganjali M.A., Ghodsi A., Parvizi S., On type IIA string theory on the PP-wave background, Nuclear Phys. B, 2003, V.661, 174-190, 2003, hep-th/0207037.

[5] Alishahiha M., Kumar A., D-brane solutions from new isometries of pp-waves, Phys. Lett. B, 2002, V.542, 130-136, hep-th/0205134.

[6] Atiyah M.F., Hitchin N.J., Singer I.M., Self-duality in four-dimensional Riemannian geometry. Proc. Roy. Soc. London Ser. A, 1978, V.362, N 1711, 425-461.

[7] Bär C., Real Killing spinors and holonomy, Comm. Math. Phys., 1993, V.154, 509-521.

[8] Baum H., Complete Riemannian manifolds with imaginary Killing spinors, Ann. Global Anal. Geom., 1989, V.7, 205-226.

[9] Baum H., Friedrich T., Grunewald R., Kath I., Twistors and Killing spinors on Riemannian manifolds, Teubner-Texte zur Mathematik, Vol. 124, Stuttgart, B.G. Teubner Verlagsgesellschaft, 1991.

[10] Bergshoeff E., de Roo M., Janssen B., Ortín T., The super D9-brane and its truncations, Nuclear Phys. B, 1999, V.550, 289-302, 1999, hep-th/9901055.

[11] Bernhardt N., Nagy P.-A., On algebraic torsion forms and their spin holonomy algebra, math.DG/0608509.

[12] Blau M., Supergravity solitons, Lecture available at http://www.unine.ch/phys/string/mblau/mblau.html.

[13] Chevalley C., The algebraic theory of spinors and Clifford algebras, Collected works, Vol. 2, Berlin, SpringerVerlag, 1997 (Edited and with a foreword by P. Cartier and C. Chevalley, postface by J.-P. Bourguignon).

[14] Cremmer E., Julia B., The SO(8) supergravity, Nuclear Phys. B, 1979, V.159, 141-212.

[15] Cremmer E., Julia B., Scherk J., Supergravity theory in 11 dimensions, Phys. Lett. B, 1979, V.76, 409-412.

[16] Friedrich T., Stefan Ivanov S., Parallel spinors and connections with skew-symmetric torsion in string theory, Asian J. Math., 2002, V.6, 303-335, math.DG/0102142.

[17] Green M.B., Schwarz J.H., Witten E., Superstring theory, Vols. 1-2, 2nd ed., Cambridge Monographs on Mathematical Physics, Cambridge, Cambridge University Press, 1988.

[18] Ivanov P., Ivanov S., SU(3)-instantons and $G_{2}$, Spin(7)-heterotic string solitons, Comm. Math. Phys., 2005, V.259, 79-102, math.DG/0312094.

[19] Ivanov S., Connections with torsion, parallel spinors and geometry of Spin(7) manifolds, Math. Res. Lett., 2004, V.11, 171-186, math.DG/0111216.

[20] Ivanov S., Papadopoulos G., Vanishing theorems and string backgrounds, Class. Quant. Grav., 2001, V.18, 1089-1110, math.DG/0010038. 
[21] Kath I., Killing spinors on pseudo-Riemannian manifolds, Habilitation Thesis, Berlin, Humboldt University, 2000 .

[22] Kath I., Parallel pure spinors on pseudo-Riemannian manifolds, in Geometry and Topology of Submanifolds X, Proceedings of the Conference on Differential Geometry in Honor of Prof. S.S. Chern (1999, Beijing Berlin), Editors W.H. Chen et al., Singapore, World Scientific, 2000, 87-103.

[23] Kennedy A.D., Clifford algebras in 2 $\omega$ dimensions, J. Math. Phys, 1981, V.22, 1330-1337.

[24] Klinker F., Supersymmetric Killing structures, PhD Thesis, University Leipzig, 2003.

[25] Klinker F., Supersymmetric Killing structures, Comm. Math. Phys., 2005, V.255, 419-467.

[26] Kostant B., Graded manifolds, graded Lie theory, and prequantization. in Differential Geometrical Methods in Mathematical Physics (1975, University of Bonn), Lecture Notes in Math., Vol. 570, Berlin, Springer, Berlin, 1977, 177-306.

[27] Lawson H.B.Jr., Michelsohn M.-L., Spin geometry, Princeton Mathematical Series, Vol. 38, Princeton, NJ, Princeton University Press, 1989.

[28] Mangiarotti L., Sardanashvily G., Connections in classical and quantum field theory, Singapore, World Scientific, 2000.

[29] Moroianu A., On the infinitesimal isometries of manifolds with Killing spinors, J. Geom. Phys., 2000, V.35, $63-74$.

[30] Papadopoulos G., Spin cohomology, J. Geom. Phys., 2006, V.56, 1893-1919, math.DG/0410494.

[31] Papadopoulos G., Tsimpis D., The holonomy of the supercovariant connection and Killing spinors, JHEP, 2003, V.7, 018, 28 pages, hep-th/0306117.

[32] van Proeyen A., Tools for supersymmetry, hep-th/9910030. 